\title{
On the failure of the Gorenstein property for Hecke algebras of prime weight
}

\author{
L. J. P. Kilford and Gabor Wiese
}

16th August 2018

\begin{abstract}
In this article we report on extensive calculations concerning the Gorenstein defect for Hecke algebras of spaces of modular forms of prime weight $p$ at maximal ideals of residue characteristic $p$ such that the attached $\bmod p$ Galois representation is unramified at $p$ and the Frobenius at $p$ acts by scalars. The results lead us to the ask the question whether the Gorenstein defect and the multplicity of the attached Galois representation are always equal to 2 . We review the literature on the failure of the Gorenstein property and multiplicity one, discuss in some detail a very important practical improvement of the modular symbols algorithm over finite fields and include precise statements on the relationship between the Gorenstein defect and the multiplicity of Galois representations.
\end{abstract}

MSC Classification: 11F80 (primary), 11F33, 11F25 (secondary).

\section{Contents}

\section{Introduction}

1.1 Previous results on the failure of multiplicity one or the Gorenstein property . . . . .

1.2 New results . . . . . . . . . . . . . . . . . . . .

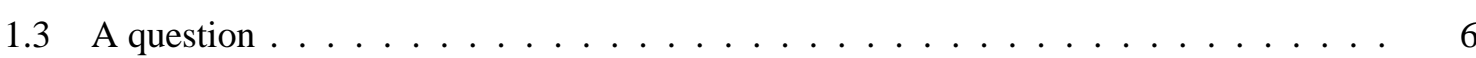

2 Relation between multiplicity and Gorenstein defect

3 Modular Symbols and Hecke Algebras 10

3.1 Mod $p$ modular forms and modular symbols . . . . . . . . . . . . . . 10

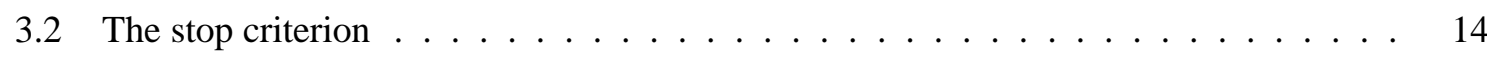

3.3 Algorithm . . . . . . . . . . . . . . . . . . 17

4 Computational results 18

4.1 Table entries . . . . . . . . . . . . . . . . . . . . . . 19

4.2 Dihedral examples . . . . . . . . . . . . . . . . . . . . . . . . . . . . . . . . . .

4.3 Icosahedral example . . . . . . . . . . . . . . . . . . 20 
6 Acknowledgements 23

A The Magma package HeckeAlgebra (by Gabor Wiese) 26

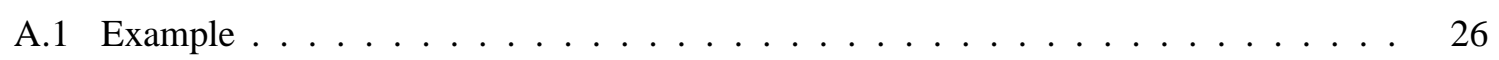

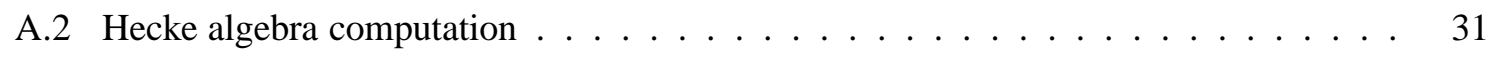

A.2.1 The modular form format . . . . . . . . . . . . . . 31

A.2.2 Dihedral modular forms . . . . . . . . . . . . 32

A.2.3 Icosahedral modular forms . . . . . . . . . . . . . . . . . 33

A.2.4 The Hecke algebra format $\ldots \ldots \ldots \ldots \ldots \ldots$

A.2.5 Hecke algebras . . . . . . . . . . . . . . . . . . . . 34

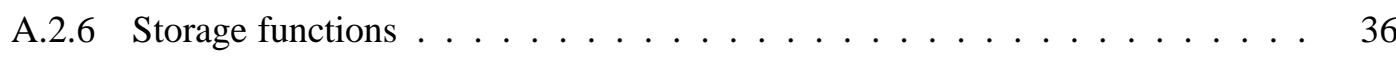

A.2.7 Output functions $\ldots \ldots \ldots \ldots \ldots \ldots \ldots \ldots \ldots$

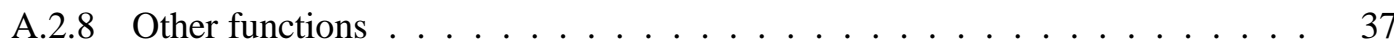

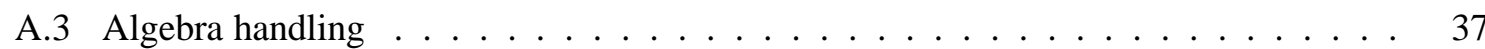

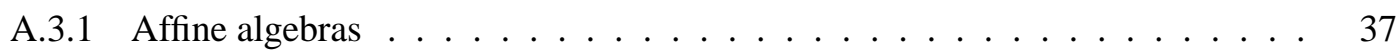

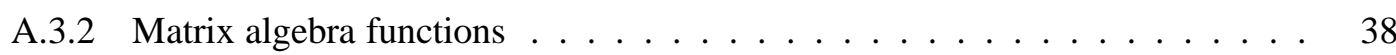

A.3.3 Associative algebras . . . . . . . . . . . . . . . . . . . 40

A.3.4 Gorenstein defect . . . . . . . . . . . . . . . . . 40

B Tables of Hecke algebras

\section{Introduction}

In Wiles' proof of Fermat's Last Theorem (see [24]) an essential step was to show that certain Hecke algebras are Gorenstein rings. Moreover, the Gorenstein property of Hecke algebras is equivalent to the fact that Galois representations appear on certain Jacobians of modular curves precisely with multiplicity one. This article is concerned with the Gorenstein property and with the multiplicity one question. We report previous work and exhibit many new examples where multiplicity one and the Gorenstein property fail. We compute the multiplicty in these cases. Moreover, we ask the question suggested by our computations whether it is always equal to two if it fails.

We first have to introduce some notation. For integers $N \geq 1$ and $k \geq 2$ and a Dirichlet character $\chi:(\mathbb{Z} / N \mathbb{Z})^{\times} \rightarrow \mathbb{C}^{\times}$we let $S_{k}\left(\Gamma_{1}(N)\right)$ be the $\mathbb{C}$-vector space of holomorphic cusp forms on $\Gamma_{1}(N)$ of weight $k$ and $S_{k}(N, \chi)$ the subspace on which the diamond operators act through the character $\chi$. We now introduce some extra notation for Hecke algebras over specified rings.

Notation 1.1 (Notation for Hecke algebras) Whenever $S \subseteq R$ are rings and $M$ is an $R$-module on which the Hecke and diamond operators act, we let $\mathbb{T}_{S}(M)$ be the $S$-subalgebra inside the $R$ - 
endomorphism ring of $M$ generated by the Hecke and the diamond operators. If $\phi: S \rightarrow S^{\prime}$ is a ring homomorphism, we let $\mathbb{T}_{\phi}(M):=\mathbb{T}_{S}(M) \otimes_{S} S^{\prime}$ or with $\phi$ understood $\mathbb{T}_{S \rightarrow S^{\prime}}(M)$.

We will mostly be dealing with the Hecke algebras $\mathbb{T}_{\mathbb{Z}}\left(S_{k}\left(\Gamma_{1}(N)\right)\right)$ and $\mathbb{T}_{\mathbb{Z}[\chi]}\left(S_{k}(N, \chi)\right)$, their completions $\mathbb{T}_{\mathbb{Z} \rightarrow \mathbb{Z}_{p}}\left(S_{k}\left(\Gamma_{1}(N)\right)\right)$ and $\mathbb{T}_{\mathcal{O} \rightarrow \widehat{\mathcal{O}}}\left(S_{k}(N, \chi)\right)$, as well as their reductions $\mathbb{T}_{\mathbb{Z} \rightarrow \mathbb{F}_{p}}\left(S_{k}\left(\Gamma_{1}(N)\right)\right)$ and $\mathbb{T}_{\mathcal{O} \rightarrow \mathbb{F}}\left(S_{k}(N, \chi)\right)$. Here, $p$ is a prime and $\mathcal{O}=\mathbb{Z}[\chi]$ is the smallest subring of $\mathbb{C}$ containing all values of $\chi, \widehat{\mathcal{O}}$ is a completion and $\mathcal{O} \rightarrow \mathbb{F}$ is a reduction modulo a prime above $p$. In Section 3 the reduced Hecke algebras are identified with Hecke algebras of $\bmod p$ modular forms, which are closely related to Hecke algebras of Katz modular forms over finite fields (see Section 2).

We choose a holomorphic cuspidal Hecke eigenform as the starting point of our discussion and treatment. So let $f \in S_{k}(N, \chi) \subseteq S_{k}\left(\Gamma_{1}(N)\right)$ be an eigenform for all Hecke and diamond operators. It (more precisely, its Galois conjugacy class) corresponds to minimal ideals, both denoted by $\mathfrak{p}_{f}$, in each of the two Hecke algebras $\mathbb{T}_{\mathbb{Z}}\left(S_{k}\left(\Gamma_{1}(N)\right)\right)$ and $\mathbb{T}_{\mathbb{Z}[\chi]}\left(S_{k}(N, \chi)\right)$. We also choose maximal ideals $\mathfrak{m}=\mathfrak{m}_{f}$ containing $\mathfrak{p}_{f}$ of residue characteristic $p$ again in each of the two. Note that the residue fields are the same in both cases.

By work of Shimura and Deligne, one can associate to $f$ (more precisely, to $\mathfrak{m}$ ) a continuous odd semi-simple Galois representation

$$
\rho_{f}=\rho_{\mathfrak{m}_{f}}=\rho_{\mathfrak{m}}: \operatorname{Gal}(\overline{\mathbb{Q}} / \mathbb{Q}) \rightarrow \operatorname{GL}_{2}\left(\mathbb{T}_{\mathbb{Z}}\left(S_{k}(N, \chi)\right) / \mathfrak{m}\right)
$$

unramified outside $N p$ satisfying $\operatorname{Tr}\left(\rho_{\mathfrak{m}}\left(\operatorname{Frob}_{l}\right)\right) \equiv T_{l} \bmod \mathfrak{m}$ and $\operatorname{Det}\left(\rho_{m}\left(\operatorname{Frob}_{l}\right)\right) \equiv l^{k-1} \chi(l)$ $\bmod \mathfrak{m}$ for all primes $l \nmid N p$. In the case of weight $k=2$ and level $N$, the representation $\rho_{m}$ can be naturally realised on the $p$-torsion points of the Jacobian of the modular curve $X_{1}(N)_{\mathbb{Q}}$. The algebra $\mathbb{T}_{\mathbb{Z} \rightarrow \mathbb{F}_{p}}\left(S_{2}\left(\Gamma_{1}(N)\right)\right)$ acts naturally on $J_{1}(N)_{\mathbb{Q}}(\overline{\mathbb{Q}})[p]$ and we can form the Galois module $J_{1}(N)_{\mathbb{Q}}(\overline{\mathbb{Q}})[\mathfrak{m}]=J_{1}(N)_{\mathbb{Q}}(\overline{\mathbb{Q}})[p][\widetilde{\mathfrak{m}}]$ with $\widetilde{\mathfrak{m}}$ the maximal ideal of $\mathbb{T}_{\mathbb{Z} \rightarrow \mathbb{F}_{p}}\left(S_{2}\left(\Gamma_{1}(N)\right)\right)$ which is the image of $\mathfrak{m}$ under the natural projection. Supposing that $\rho_{m}$ is absolutely irreducible, the main result of [3] shows that the Galois representation $J_{1}(N p)_{\mathbb{Q}}(\overline{\mathbb{Q}})[\mathfrak{m}]$ is isomorphic to a direct sum of $r$ copies of $\rho_{m}$ for some integer $r \geq 1$, which one calls the multiplicity of $\rho_{m}$ (cf. [18]). One says that $\rho_{m}$ is a multiplicity one representation or satisfies multiplicity one, if $r=1$. See [16] for a similar definition for $J_{0}(N)$ and Prop. 2.6 for a comparison.

The notion of multiplicity can be naturally extended to Galois representations attached to eigenforms $f$ of weights $3 \leq k \leq p+1$ for $p \nmid N$. This is accomplished by a result of Serre's which implies the existence of a maximal ideal $\mathfrak{m}_{2} \subset \mathbb{T}_{\mathbb{Z}}\left(S_{2}\left(\Gamma_{1}(N p)\right)\right.$ ) such that $\rho_{\mathfrak{m}_{f}} \cong \rho_{\mathfrak{m}_{2}}$ (see Prop. 2.3). One hence obtains the notion of multiplicity (on $J_{1}(N p)$ ) for the representation $\rho_{m_{f}}$ by defining it as the multiplicity of $\rho_{\mathfrak{m}_{2}}$. Moreover, when allowing twists by the cyclotomic character, it is even possible to treat arbitrary weights. The following theorem summarises results on when the multiplicity in the above sense is known to be one.

Theorem 1.2 (Mazur [16], Edixhoven [7], Gross [13], Buzzard [5]) Let $\rho_{\mathfrak{m}}$ be a representation associated with a modular cuspidal eigenform $f \in S_{k}(N, \chi)$ and let $p$ be the residue characteristic of $\mathfrak{m}$. Suppose that $\rho_{\mathfrak{m}}$ is absolutely irreducible and that $p$ does not divide $N$. If either 
1. $2 \leq k \leq p-1$, or

2. $k=p$ and $\rho_{\mathfrak{m}}$ is ramified at $p$, or

3. $k=p$ and $\rho_{\mathfrak{m}}$ is unramified at $p$ and $\rho_{\mathfrak{m}}\left(\right.$ Frob $\left._{p}\right)$ is not scalar,

then the multiplicity of $\rho_{\mathfrak{m}}$ is one.

This theorem is composed of Lemma 15.1 from Mazur [16], Theorem 9.2 from Edixhoven [7], Proposition 12.10 from Gross [13] and Theorem 6.1 from Buzzard [5].

The following theorem by the second author ([23], Corollary 4.4) tells us when the multiplicity is not one.

Theorem 1.3 Let $\rho_{\mathfrak{m}}$ as in the previous theorem. Suppose $k=p$ and that $\rho_{\mathfrak{m}}$ is unramified at $p$. If $p=2$, assume also that a Katz cusp form over $\mathbb{F}_{2}$ of weight 1 on $\Gamma_{1}(N)$ exists which gives rise to $\rho_{\mathfrak{m}}$.

If $\rho_{\mathfrak{m}}\left(\right.$ Frob $\left._{p}\right)$ is a scalar matrix, then the multiplicity of $\rho_{\mathfrak{m}}$ is bigger than 1.

In Section 2 we explain how the Galois representation $J_{1}(N p)_{\mathbb{Q}}(\overline{\mathbb{Q}})[\mathfrak{m}]$ is related to the different Hecke algebras evoked above and see in many cases of interest a precise relationship between the geometrically defined term of multiplicity and the Gorenstein defect of these algebras. The latter can be computed explicitly, which is the subject of the present article. We now give the relevant definitions.

Definition 1.4 (The Gorenstein property) Let $A$ be a local Noetherian ring with maximal ideal $\mathfrak{m}$. Suppose first that the Krull dimension of $A$ is zero, i.e. that $A$ is Artinian. We then define the Gorenstein defect of $A$ to be the minimum number of A-module generators of the annihilator of $\mathfrak{m}$ (i.e. $A[\mathfrak{m}]$ ) minus 1; equivalently, this is the $A / \mathfrak{m}$-dimension of the annihilator of $\mathfrak{m}$ minus 1 . We say that $A$ is Gorenstein if its Gorenstein defect is 0 , and non-Gorenstein otherwise. If the Krull dimension of $A$ is positive, we inductively call $A$ Gorenstein, if there exists a non-zero-divisor $x \in \mathfrak{m}$ such that $A /(x)$ is a Gorenstein ring of smaller Krull dimension (see [10], p. 532; note that our definition implies that $A$ is Cohen-Macaulay). A (not necessarily local) Noetherian ring is said to be Gorenstein if and only if all of its localisations at its maximal ideals are Gorenstein.

We will for example be interested in the Gorenstein property of $\mathbb{T}_{\mathbb{Z}}\left(S_{k}\left(\Gamma_{1}(N)\right)\right)_{\mathfrak{m}}$. Choosing $x=p$ in the definition, we see that this is equivalent to the Gorenstein defect of the finite dimensional $\mathbb{F}_{p}$-algebra $\mathbb{T}_{\mathbb{Z} \rightarrow \mathbb{F}_{p}}\left(S_{k}\left(\Gamma_{1}(N)\right)\right)_{\mathfrak{m}}$ being zero. Whenever we refer to the Gorenstein defect of the former algebra (over $\mathbb{Z}$ ), we mean the one of the latter. Our computations will concern the Gorenstein defect of $\mathbb{T}_{\mathcal{O} \rightarrow \mathbb{F}}\left(S_{k}\left(\Gamma_{1}(N), \chi\right)\right)_{\mathfrak{m}}$. See Section 2 for a comparison with the one not involving a character. It is important to remark that the Gorenstein defect of a local Artin algebra over a field does not change after passing to a field extension and taking any of the conjugate local factors.

We illustrate the definition by an example. The algebra $k[x, y, z] /\left(x^{2}, y^{2}, z^{2}, x y, x z, y z\right)$ for a field $k$ is Artinian and local with maximal ideal $\mathfrak{m}:=(x, y, z)$ and the annihilator of $\mathfrak{m}$ is $\mathfrak{m}$ itself, so the Gorenstein defect is $3-1=2$. We note that this particular case does occur in nature; a 
localisation $\mathbb{T}_{\mathbb{Z} \rightarrow \mathbb{F}_{2}}\left(S_{2}\left(\Gamma_{0}(431)\right)\right)_{\mathfrak{m}}$ at one maximal ideal is isomorphic to this, with $k=\mathbb{F}_{2}$ (see [11], the discussion just before Lemma 6.6). This example can also be verified with the algorithm presented in this paper.

We now state a translation of Theorem 1.2 in terms of Gorenstein defects, which is immediate from the propositions in Section 2

Theorem 1.5 Assume the set-up of Theorem 1.2 and that one of 1., 2., or 3. is satisfied. We use notations as in the discussion of multiplicities above.

If $k=2$, then $\mathbb{T}_{\mathbb{Z}}\left(S_{2}\left(\Gamma_{1}(N)\right)\right)_{\mathfrak{m}}$ is a Gorenstein ring.

If $k \geq 3$, then $\mathbb{T}_{\mathbb{Z}}\left(S_{2}\left(\Gamma_{1}(N p)\right)\right)_{\mathfrak{m}_{2}}$ is, too. Supposing in addition that $\mathfrak{m}$ is ordinary (i.e. $T_{p} \notin \mathfrak{m}$ ), then also $\mathbb{T}_{\mathbb{Z}}\left(S_{k}\left(\Gamma_{1}(N)\right)\right)_{\mathfrak{m}}$ is Gorenstein. If, moreover, $p \geq 5$ or $\rho_{\mathfrak{m}}$ is not induced from $\mathbb{Q}(\sqrt{-1})$ (if $p=2$ ) or $\mathbb{Q}(\sqrt{-3})$ (if $p=3$ ), then $\mathbb{T}_{\mathcal{O} \rightarrow \mathbb{F}}\left(S_{k}(N, \chi)\right)_{\mathfrak{m}}$ is Gorenstein as well.

We now turn our attention to computing the Gorenstein defect and the multiplicity in the case when it is known not to be one.

Corollary 1.6 Let $\rho_{\mathfrak{m}}$ be a representation associated with a cuspidal eigenform $f \in S_{p}(N, \chi)$ with $p$ the residue characteristic of $\mathfrak{m}$. Assume that $\rho_{\mathfrak{m}}$ is absolutely irreducible, unramified at $p$ such that $\rho_{\mathfrak{m}}\left(\right.$ Frob $\left._{p}\right)$ is a scalar matrix. Let $r$ be the multiplicity of $\rho_{\mathfrak{m}}$ and $d$ the Gorenstein defect of any of $\mathbb{T}_{\mathcal{O} \rightarrow \mathbb{F}}\left(S_{k}(N, \chi)\right)_{\mathfrak{m}}, \mathbb{T}_{\mathbb{Z} \rightarrow \mathbb{F}_{p}}\left(S_{k}\left(\Gamma_{1}(N)\right)\right)_{\mathfrak{m}}$ or $\mathbb{T}_{\mathbb{Z} \rightarrow \mathbb{F}_{p}}\left(S_{2}\left(\Gamma_{1}(N p)\right)\right)_{\mathfrak{m}_{2}}$

Then the relation $d=2 r-2$ holds.

Proof. The equality of the Gorenstein defects and the relation with the multiplicity are proved in Section 2] noting that $\mathfrak{m}$ is ordinary, as $a_{p}(f)^{2}=\chi(p) \neq 0$ by [13], p. 487 .

\subsection{Previous results on the failure of multiplicity one or the Gorenstein property}

Prior to the present work and the article [23], there have been some investigations into when Hecke algebras fail to be Gorenstein. In [14], the first author showed, using MAGMA [2], that the Hecke algebras $\mathbb{T}_{\mathbb{Z}}\left(S_{2}\left(431, \chi_{\text {triv }}\right)\right), \mathbb{T}_{\mathbb{Z}}\left(S_{2}\left(503, \chi_{\text {triv }}\right)\right)$ and $\mathbb{T}_{\mathbb{Z}}\left(S_{2}\left(2089, \chi_{\text {triv }}\right)\right)$ are not Gorenstein by explicit computation of the localisation of the Hecke algebra at a suitable maximal ideal above 2, and in [18], it is shown that $\mathbb{T}_{\mathbb{Z}}\left(S_{2}\left(2071, \chi_{\text {triv }}\right)\right)$ is not Gorenstein in a similar fashion. These examples were discovered by considering elliptic curves $E / \mathbb{Q}$ such that in the ring of integers of $\mathbb{Q}(E[2])$ the prime ideal (2) splits completely, and then doing computations with MAGMA.

There are also some results in the literature on the failure of multiplicity one within the torsion of certain Jacobians. In [1], the following theorem is proved:

Theorem 1.7 (Agashe-Ribet-Stein [1], Proposition 5.1) Suppose that $E$ is an optimal elliptic curve over $\mathbb{Q}$ of conductor $N$, with congruence number $r_{E}$ and modular degree $m_{E}$ and that $p$ is a prime such that $p \mid r_{E}$ but $p \nmid m_{E}$. Let $\mathfrak{m}$ be the annihilator in $\mathbb{T}_{\mathbb{Z}}\left(S_{2}\left(N, \chi_{\text {triv }}\right)\right)$ of $E[p]$. Then multiplicity one fails for $\mathfrak{m}$. 
They give a table of examples; for instance, $\mathbb{T}_{\mathbb{Z}}\left(S_{2}\left(54, \chi_{\text {triv }}\right)\right)$ does not satisfy multiplicity one at some maximal ideal above 3 . It is not clear whether this phenomenon occurs infinitely often.

In [17], it is shown that the $\bmod p$ multiplicity of a certain representation in the Jacobian of the Shimura curve derived from the rational quaternion algebra of discriminant $11 \cdot 193$ is 2 ; this result inspired the calculations in [14].

Let us finally mention that for $p=2$ precisely the Galois representations $\rho$ with image equal to the dihedral group $D_{3}$ come from an elliptic curve over $\mathbb{Q}$. For, on the one hand, we only need observe that $D_{3}=\mathrm{GL}_{2}\left(\mathbb{F}_{2}\right)$. On the other hand, any $S_{3}$-extension $K$ of the rationals can be obtained as the splitting field of an irreducible integral polynomial $f=X^{3}+a X+b$. The 2-torsion of the elliptic curve $E: Y^{2}=f$ consists precisely of the three roots of $f$ and the point at infinity. So, the field generated over $\mathbb{Q}$ by the 2-torsion of $E$ is $K$.

\subsection{New results}

Using the modular symbols algorithm over finite fields with an improved stop criterion (see Section 3), we performed computations in MAGMA concerning the Gorenstein defect of Hecke algebras of cuspidal modular forms of prime weights $p$ at maximal ideals of residue characteristic $p$ in the case of Theorem 1.3. All of our 384 examples have Gorenstein defect equal to 2 and hence their multiplicity is 2 .

We formulate part of our computational findings as a theorem.

Theorem 1.8 For every prime $p<100$ there exists a prime $N \neq p$ and a Dirichlet character $\chi$ such that the Hecke algebra $\mathbb{T}_{\mathbb{Z}[\chi] \rightarrow \mathbb{F}}\left(S_{p}(N, \chi)\right)$ has Gorenstein defect 2 at some maximal ideal $\mathfrak{m}$ of residue characteristic $p$. The corresponding Galois representation $\rho_{\mathfrak{m}}$ appears with multiplicity two on the p-torsion of the Jacobian $J_{1}(N p)_{\mathbb{Q}}(\overline{\mathbb{Q}})$ if $p$ is odd, respectively $J_{1}(N)_{\mathbb{Q}}(\overline{\mathbb{Q}})$ if $p=2$.

Our computational results are discussed in more detail in Section 4 .

\subsection{A question}

Question 1.9 Let $p$ be a prime. Let $f$ be a normalised cuspidal modular eigenform of weight $p$, prime level $N \neq p$ for some Dirichlet character $\chi$. Let $\rho_{f}: G_{\mathbb{Q}} \rightarrow \mathrm{GL}_{2}\left(\overline{\mathbb{F}}_{p}\right)$ be the modular Galois representation attached to $f$. We assume that $\rho_{f}$ is irreducible and unramified at $p$ and that $\rho_{f}\left(\operatorname{Frob}_{p}\right)$ is a scalar matrix.

Write $\mathbb{T}_{\mathbb{F}}$ for $\mathbb{T}_{\mathbb{Z}[\chi] \rightarrow \mathbb{F}}\left(S_{p}(N, \chi)\right)$. Recall that this notation stands for the tensor product over $\mathbb{Z}[\chi]$ of a residue field $\mathbb{F} / \mathbb{F}_{p}$ of $\mathbb{Z}[\chi]$ by the $\mathbb{Z}[\chi]$-algebra generated inside the endomorphism algebra of $S_{p}(N, \chi)$ by the Hecke operators and by the diamond operators. Let $\mathfrak{m}$ be the maximal ideal of $\mathbb{T}_{\mathbb{F}}$ corresponding to $f$.

Is the Gorenstein defect of the Hecke algebra $\mathbb{T}_{\mathbb{F}}$ localised at $\mathfrak{m}$, denoted by $\mathbb{T}_{\mathfrak{m}}$, always equal to 2 ?

Equivalently, is the multiplicity of the Galois representation attached to $f$ always equal to 2 ? 
This question was also raised both by Kevin Buzzard and James Parson in communications to the authors.

\section{Relation between multiplicity and Gorenstein defect}

In this section we collect results, some of which are well-known, on the multiplicity of Galois representations, the Gorenstein defect and relations between the two. Whereas the mod $p$ modular symbols algorithm naturally computes mod $p$ modular forms (see Section 3), this rather geometrical section uses (mostly in the references) the theory of Katz modular forms over finite fields (see e.g. [8]). If $N \geq 5$ and $k \geq 2$, the Hecke algebra $\mathbb{T}_{\mathbb{Z} \rightarrow \mathbb{F}_{p}}\left(S_{k}\left(\Gamma_{1}(N)\right)\right)$ is both the Hecke algebra of mod $p$ cusp forms of weight $k$ on $\Gamma_{1}(N)$ and the Hecke algebra of the corresponding Katz cusp forms over $\mathbb{F}_{p}$. However, in the presence of a Dirichlet character $\mathbb{T}_{\mathbb{Z}[\chi] \rightarrow \mathbb{F}}\left(S_{k}(N, \chi)\right)$ only has an interpretation as the Hecke algebra of the corresponding mod $p$ cusp forms and there may be differences with the respective Hecke algebra for Katz forms (see Carayol's Lemma, Prop. 1.10 of [8]).

We start with the well-known result in weight 2 (see e.g. [16], Lemma 15.1) that multiplicity one implies that the corresponding local Hecke factor is a Gorenstein ring.

Proposition 2.1 Let $\mathfrak{m}$ be a maximal ideal of $\mathbb{T}:=\mathbb{T}_{\mathbb{Z}}\left(S_{2}\left(\Gamma_{1}(N)\right)\right)$ of residue characteristic $p$ which may divide $N$. Denote by $\widetilde{\mathfrak{m}}$ the image of $\mathfrak{m}$ in $\mathbb{T}_{\mathbb{F}_{p}}:=\mathbb{T} \otimes_{\mathbb{Z}} \mathbb{F}_{p}=\mathbb{T}_{\mathbb{Z} \rightarrow \mathbb{F}_{p}}\left(S_{2}\left(\Gamma_{1}(N)\right)\right)$. Suppose that the Galois representation $\rho_{\mathfrak{m}}$ is irreducible and satisfies multiplicity one (see Section प).

Then as $\mathbb{T}_{\mathbb{F}_{p}, \widetilde{\mathfrak{m}}}$-modules one has

$$
J_{1}(N)_{\mathbb{Q}}(\overline{\mathbb{Q}})[p]_{\tilde{\mathfrak{m}}} \cong \mathbb{T}_{\mathbb{F}_{p}, \tilde{\mathfrak{m}}} \oplus \mathbb{T}_{\mathbb{F}_{p}, \tilde{\mathfrak{m}}}
$$

and the localisations $\mathbb{T}_{\mathfrak{m}}$ and $\mathbb{T}_{\mathbb{F}_{p}, \widetilde{\mathfrak{m}}}$ are Gorenstein rings. Similar results hold if one replaces $\Gamma_{1}(N)$ and $J_{1}(N)$ by $\Gamma_{0}(N)$ and $J_{0}(N)$.

Proof. For the proof we have to pass to $\mathbb{T}_{\mathbb{Z}_{p}}=\mathbb{T}_{\mathbb{Z}} \otimes_{\mathbb{Z}} \mathbb{Z}_{p}$. We also denote by $\mathfrak{m}$ the maximal ideal in $\mathbb{T}_{\mathbb{Z}_{p}}$ that corresponds to $\mathfrak{m}$. Let $V$ be the $\mathfrak{m}$-part of the $p$-Tate module of $J_{1}(N)_{\mathbb{Q}}$. Multiplicity one implies that $V / \mathfrak{m} V$ is a 2-dimensional $\mathbb{T} / \mathfrak{m}=\mathbb{T}_{\mathbb{Z}_{p}} / \mathfrak{m}=\mathbb{T}_{\mathbb{F}_{p}, \widetilde{\mathfrak{m}}} / \widetilde{\mathfrak{m}}$-vector space, since

$$
V / \mathfrak{m} V \cong(V / p V) / \widetilde{\mathfrak{m}} \cong\left(J_{1}(N)_{\mathbb{Q}}(\overline{\mathbb{Q}})[p]\right) / \widetilde{\mathfrak{m}} \cong\left(J_{1}(N)_{\mathbb{Q}}(\overline{\mathbb{Q}})[p]\right)^{\vee} / \widetilde{\mathfrak{m}} \cong\left(J_{1}(N)_{\mathbb{Q}}(\overline{\mathbb{Q}})[\mathfrak{m}]\right)^{\vee}
$$

where the self-duality comes from the modified Weil pairing which respects the Hecke action (see

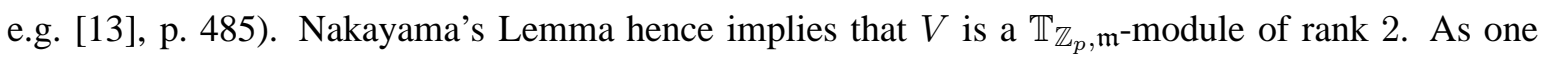
knows that $V \otimes_{\mathbb{Z}_{p}} \mathbb{Q}_{p}$ is a $\mathbb{T}_{\mathfrak{m}} \otimes \mathbb{Q}_{p}$-module of rank 2 , it follows that $V$ is a free $\mathbb{T}_{\mathbb{Z}_{p}, \mathfrak{m} \text {-module }}$

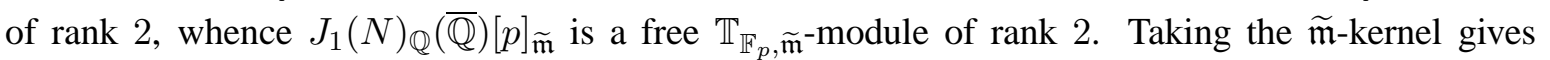
$J_{1}(N)_{\mathbb{Q}}(\overline{\mathbb{Q}})[\mathfrak{m}]=\left(\mathbb{T}_{\mathbb{F}_{p}, \widetilde{\mathfrak{m}}}[\widetilde{\mathfrak{m}}]\right)^{2}$, whence the Gorenstein defect is zero. In the $\Gamma_{0}$-situation, the same proof holds.

In the so-called ordinary case, we have the following precise relationship between the multiplicity and the Gorenstein defect, which was suggested to us by Kevin Buzzard. The proof can be found in [23]. 
Proposition 2.2 Suppose $p \nmid N$ and let $M=N$ or $M=N p$. Let $\mathfrak{m}$ be a maximal ideal of $\mathbb{T}_{\mathbb{Z}}\left(S_{2}\left(\Gamma_{1}(M)\right)\right)$ of residue characteristic $p$ and assume that $\mathfrak{m}$ is ordinary, i.e. that the $p$-th Hecke operator $T_{p}$ is not in $\mathfrak{m}$. Assume also that $\rho_{\mathfrak{m}}$ is irreducible. Denote by $\widetilde{\mathfrak{m}}$ the image of $\mathfrak{m}$ in $\mathbb{T}_{\mathbb{F}_{p}}:=\mathbb{T}_{\mathbb{Z} \rightarrow \mathbb{F}_{p}}\left(S_{2}\left(\Gamma_{1}(M)\right)\right)$. Then the following statements hold:

(a) There is the exact sequence

$$
0 \rightarrow \mathbb{T}_{\mathbb{F}_{p}, \widetilde{\mathfrak{m}}} \rightarrow J_{1}(M)(\overline{\mathbb{Q}})[p]_{\tilde{\mathfrak{m}}} \rightarrow \mathbb{T}_{\mathbb{F}_{p}, \widetilde{\mathfrak{m}}}^{\vee} \rightarrow 0
$$

of $\mathbb{T}_{\mathbb{F}_{p}, \widetilde{\mathfrak{m}}}$-modules, where the dual is the $\mathbb{F}_{p}$-linear dual.

(b) If $d$ is the Gorenstein defect of $\mathbb{T}_{\mathbb{F}_{p}, \widetilde{\mathfrak{m}}}$ and $r$ is the multiplicity of $\rho_{\mathfrak{m}}$, then the relation

$$
d=2 r-2
$$

holds.

We now establish a relation between $\bmod p$ Hecke algebras of weights $3 \leq k \leq p+1$ for levels $N$ not divisible by $p$ with Hecke algebras of weight 2 and level $N p$. It is needed in order to compare the Hecke algebras in higher weight to those acting on the $p$-torsion of Jacobians and thus to make a link to the multiplicity of the attached Galois representations.

Proposition 2.3 Let $N \geq 5, p \nmid N$ and $3 \leq k \leq p+1$. Let $\mathfrak{m}$ be a maximal ideal of the mod $p$ Hecke algebra $\mathbb{T}_{\mathbb{Z} \rightarrow \mathbb{F}_{p}}\left(S_{k}\left(\Gamma_{1}(N)\right)\right.$. Then there exists a maximal ideal $\mathfrak{m}_{2}$ of $\mathbb{T}_{\mathbb{Z} \rightarrow \mathbb{F}_{p}}\left(S_{2}\left(\Gamma_{1}(N p)\right)\right.$ and a natural surjection

$$
\mathbb{T}_{\mathbb{Z} \rightarrow \mathbb{F}_{p}}\left(S _ { 2 } ( \Gamma _ { 1 } ( N p ) ) _ { \mathfrak { m } _ { 2 } } \rightarrow \mathbb { T } _ { \mathbb { Z } \rightarrow \mathbb { F } _ { p } } \left(S_{k}\left(\Gamma_{1}(N)\right)_{\mathfrak{m}}\right.\right.
$$

If $\mathfrak{m}$ is ordinary $\left(T_{p} \notin \mathfrak{m}\right)$, this surjection is an isomorphism.

Proof. From Sections 5 and 6 of [22], whose notation we adopt for this proof, one obtains without difficulty the commutative diagram of Hecke algebras:

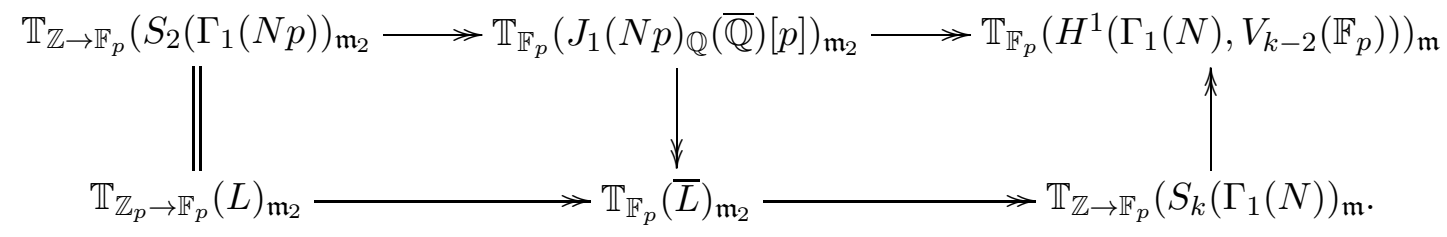

The claimed surjection can be read off. In the ordinary situation, Proposition 2.2 shows that the upper left horizontal arrow is in fact an isomorphism. That also the upper right horizontal arrow is an isomorphism is explained in [22]. The result follows immediately.

In the next proposition we compare Hecke algebras for spaces of modular forms on $\Gamma_{1}(N)$ to those of the same level and weight, but with a Dirichlet character. 
Proposition 2.4 Let $N \geq 5, k \geq 2$ and let $\chi:(\mathbb{Z} / N \mathbb{Z})^{\times} \rightarrow \mathbb{C}^{\times}$be a Dirichlet character. Let $f \in S_{k}(N, \chi) \subset S_{k}\left(\Gamma_{1}(N)\right)$ be a normalised Hecke eigenform. Let further $\mathfrak{m}_{\bar{\chi}}$ be the maximal ideal in $\mathbb{T}_{\mathbb{F}}^{\bar{\chi}}:=\mathbb{T}_{\mathbb{Z}[\chi] \rightarrow \mathbb{F}}\left(S_{k}(N, \chi)\right)$ and $\mathfrak{m}$ the one in $\mathbb{T}_{\mathbb{Z} \rightarrow \mathbb{F}_{p}}\left(S_{k}\left(\Gamma_{1}(N)\right)\right)$ of residue characteristic $p$ for $p \nmid N$ belonging to $f$. If $k=2$, suppose additionally that $\rho_{\mathfrak{m}}$ is irreducible. If $p=2$, suppose that $\rho_{\mathfrak{m}}$ is not induced from $\mathbb{Q}(\sqrt{-1})$, and if $p=3$, suppose that $\rho_{\mathfrak{m}}$ is not induced from $\mathbb{Q}(\sqrt{-3})$.

Then the Gorenstein defects of $\mathbb{T}_{\mathbb{Z}[\chi] \rightarrow \mathbb{F}}\left(S_{k}(N, \chi)\right)_{\mathfrak{m}_{\bar{\chi}}}$ and $\mathbb{T}_{\mathbb{Z} \rightarrow \mathbb{F}_{p}}\left(S_{k}\left(\Gamma_{1}(N)\right)\right)_{\mathfrak{m}}$ are equal.

Proof. Write $\Delta:=(\mathbb{Z} / N \mathbb{Z})^{\times}$and let $\Delta_{p}$ be its $p$-Sylow subgroup. Let $\bar{\chi}: \Delta \rightarrow \mathbb{F}^{\times}$be the reduction of $\chi$ obtained by composing $\chi$ with $\mathbb{Z}[\chi] \rightarrow \mathbb{F}$. As the Gorenstein defect is invariant under base extension, it is no loss to work with $\mathbb{T}_{\mathbb{F}}:=\mathbb{T}_{\mathbb{Z} \rightarrow \mathbb{F}}\left(S_{k}\left(\Gamma_{1}(N)\right)\right)$. We still write $\mathfrak{m}$ for the maximal ideal in $\mathbb{T}_{\mathbb{F}}$ belonging to $f$. Note that $\langle\delta\rangle-\bar{\chi}(\delta) \in \mathfrak{m}$ for all $\delta \in \Delta$.

We let $\Delta$ act on $\mathbb{T}_{\mathbb{F}}$ via the diamond operators and we let $\mathbb{F} \bar{\chi}$ be a copy of $\mathbb{F}$ with $\Delta$-action through the inverse of $\bar{\chi}$. We have

$$
\left(\mathbb{T}_{\mathbb{F}, \mathfrak{m}} \otimes_{\mathbb{F}} \mathbb{F}^{\bar{\chi}}\right)_{\Delta}=\left(\mathbb{T}_{\mathbb{F}, \mathfrak{m}} \otimes_{\mathbb{F}} \mathbb{F}^{\bar{\chi}}\right) /(1-\delta \mid \delta \in \Delta) \cong \mathbb{T}_{\mathbb{F}, \mathfrak{m}_{\bar{\chi}}^{\bar{\chi}}}^{\bar{\chi}}
$$

which one obtains by considering the duals, identifying Katz cusp forms with $\bmod p$ ones on $\Gamma_{1}(N)$ and applying Carayol's Lemma ([8], Prop. 1.10). For the case $k=2$, we should point the reader to the correction at the end of the introduction to [9]. However, the statement still holds after localisation at maximal ideals corresponding to irreducible representations. Moreover, the equality $\left(\mathbb{T}_{\mathbb{F}, \mathfrak{m}} \otimes_{\mathbb{F}} \mathbb{F}^{\bar{\chi}}\right)^{\Delta}=\mathbb{T}_{\mathbb{F}, \mathfrak{m}}[\langle\delta\rangle-\bar{\chi}(\delta) \mid \delta \in \Delta]$ holds by definition.

Now Lemma 7.3 of [22] tells us that the localisation at $\mathfrak{m}$ of the $\mathbb{F}$-vector space of Katz cusp forms of weight $k$ on $\Gamma_{1}(N)$ over $\mathbb{F}$ is a free $\mathbb{F}\left[\Delta_{p}\right]$-module. Note that the standing hypothesis $k \leq p+1$ of Section 7 of [22] is not used in the proof of that lemma and see also [22], Remark 7.5. From an elementary calculation one now obtains that $N_{\Delta}=\sum_{\delta \in \Delta} \delta$ induces an isomorphism

$$
\left(\mathbb{T}_{\mathbb{F}_{\mathfrak{m}}} \otimes_{\mathbb{F}} \mathbb{F}^{\bar{\chi}}\right)_{\Delta} \stackrel{N_{\Delta}}{\longrightarrow}\left(\mathbb{T}_{\mathbb{F}_{\mathfrak{m}}} \otimes_{\mathbb{F}} \mathbb{F}^{\bar{\chi}}\right)^{\Delta}
$$

We now take the $\mathfrak{m}_{\bar{\chi}}$-kernel on both sides and obtain

$$
\mathbb{T}_{\mathbb{F}, \mathfrak{m}_{\bar{\chi}}}^{\bar{\chi}}\left[\mathfrak{m}_{\bar{\chi}}\right] \cong\left(\mathbb{T}_{\mathbb{F}, \mathfrak{m}} \otimes_{\mathbb{F}} \mathbb{F}^{\bar{\chi}}\right)_{\Delta}\left[\mathfrak{m}_{\bar{\chi}}\right] \cong\left(\mathbb{T}_{\mathbb{F}, \mathfrak{m}} \otimes_{\mathbb{F}} \mathbb{F}^{\bar{\chi}}\right)_{\Delta}[\mathfrak{m}] \cong\left(\mathbb{T}_{\mathbb{F}, \mathfrak{m}} \otimes_{\mathbb{F}} \mathbb{F}^{\bar{\chi}}\right)^{\Delta}[\mathfrak{m}]=\mathbb{T}_{\mathbb{F}, \mathfrak{m}}[\mathfrak{m}]
$$

This proves that the two Gorenstein defects are indeed equal.

The Gorenstein defect that we calculate on the computer is the number $d$ of the following corollary, which relates it to the multiplicity of a Galois representation.

Corollary 2.5 Let $p$ be a prime, $N \geq 5$ an integer such that $p \nmid N, k$ an integer satisfying $2 \leq k \leq p$ and $\chi:(\mathbb{Z} / N \mathbb{Z})^{\times} \rightarrow \mathbb{C}^{\times}$a character. Let $f \in S_{k}(N, \chi)$ be a normalised Hecke eigenform. Let further $\mathfrak{m}$ denote the maximal ideal in $\mathbb{T}_{\mathbb{Z}[\chi] \rightarrow \mathbb{F}}\left(S_{k}(N, \chi)\right)$ belonging to $f$. Suppose that $\mathfrak{m}$ is ordinary and that $\rho_{\mathfrak{m}}$ is irreducible and not induced from $\mathbb{Q}(\sqrt{-1})$ (if $p=2$ ) and not induced from $\mathbb{Q}(\sqrt{-3})$ (if $p=3$ ). We define $d$ to be the Gorenstein defect of $\mathbb{T}_{\mathbb{Z}[\chi] \rightarrow \mathbb{F}}\left(S_{k}(N, \chi)\right)_{\mathfrak{m}}$ and $r$ to be the multiplicity of $\rho_{\mathfrak{m}}$.

Then the equality $d=2 r-2$ holds. 
We include the following proposition because it establishes equality of the two different notions of multiplicities of Galois representations in the case of the trivial character.

Proposition 2.6 Let $N \geq 1$ and $p \nmid N$ and $f \in S_{2}\left(\Gamma_{0}(N)\right) \subseteq S_{2}\left(\Gamma_{1}(N)\right)$ be a normalised Hecke eigenform belonging to maximal ideals $\mathfrak{m}_{0} \subseteq \mathbb{T}_{\mathbb{Z} \rightarrow \mathbb{F}_{p}}\left(S_{2}\left(\Gamma_{0}(N)\right)\right)$ and $\mathfrak{m}_{1} \subseteq \mathbb{T}_{\mathbb{Z} \rightarrow \mathbb{F}_{p}}\left(S_{2}\left(\Gamma_{1}(N)\right)\right)$ of residue characteristic $p$. Suppose that $\rho_{\mathfrak{m}_{0}} \cong \rho_{\mathfrak{m}_{1}}$ is irreducible.

Then the multiplicity of $\rho_{\mathfrak{m}_{1}}$ on $J_{1}(N)_{\mathbb{Q}}(\overline{\mathbb{Q}})[p]$ is equal to the multiplicity of $\rho_{\mathfrak{m}_{0}}$ on $J_{0}(N)_{\mathbb{Q}}(\overline{\mathbb{Q}})[p]$. Thus, if $p>2$, this multiplicity is equal to one by Theorem 1.2

Proof. Let $\Delta:=(\mathbb{Z} / N \mathbb{Z})^{\times}$. We first remark that one has the isomorphism

$$
J_{0}(N)_{\mathbb{Q}}(\overline{\mathbb{Q}})[p]_{\mathfrak{m}_{0}} \cong\left(\left(J_{1}(N)_{\mathbb{Q}}(\overline{\mathbb{Q}})[p]\right)^{\Delta}\right)_{\mathfrak{m}_{0}},
$$

which one can for example obtain by comparing with the parabolic cohomology with $\mathbb{F}_{p}$-coefficients of the modular curves $Y_{0}(N)$ and $Y_{1}(N)$. Taking the $\mathfrak{m}_{0}$-kernel yields

$$
J_{0}(N)_{\mathbb{Q}}(\overline{\mathbb{Q}})\left[\mathfrak{m}_{0}\right] \cong J_{1}(N)_{\mathbb{Q}}(\overline{\mathbb{Q}})\left[\mathfrak{m}_{1}\right],
$$

since $\mathfrak{m}_{1}$ contains $\langle\delta\rangle-1$ for all $\delta \in \Delta$.

\section{Modular Symbols and Hecke Algebras}

The aim of this section is to present the algorithm that we use for the computations of local factors of Hecke algebras of mod $p$ modular forms. It is based on $\bmod p$ modular symbols which have been implemented in MAGMA [2] by William Stein.

The bulk of this section deals with proving the main advance, namely a stop criterion (Corollary 3.8), which in practice greatly speeds up the computations in comparison with "standard" implementations, as it allows us to work with many fewer Hecke operators than indicated by the theoretical Sturm bound (Proposition 3.10). We shall list results proving that the stop criterion is attained in many cases. However, the stop criterion does not depend on them, in the sense that it being attained is equivalent to a proof that the algebra it outputs is equal to a direct factor of a Hecke algebra of mod $p$ modular forms.

Whereas for Section 2 the notion of Katz modular forms seems the right one, the present section works entirely with mod $p$ modular forms, the definition of which is also recalled. This is very natural, since all results in this section are based on a comparison with the characteristic zero theory.

\subsection{Mod $p$ modular forms and modular symbols}

\section{Mod $p$ modular forms}

Let us for the time being fix integers $N \geq 1$ and $k \geq 2$, as well as a character $\chi:(\mathbb{Z} / N \mathbb{Z})^{\times} \rightarrow \mathbb{C}^{\times}$ such that $\chi(-1)=(-1)^{k}$. Let $M_{k}(N, \chi)$ be the space of holomorphic modular forms for $\Gamma_{1}(N)$, 
Dirichlet character $\chi$, and weight $k$. It decomposes as a direct sum (orthogonal direct sum with respect to the Petersson inner product) of its cuspidal subspace $S_{k}(N, \chi)$ and its Eisenstein subspace $\operatorname{Eis}_{k}(N, \chi)$. As before, we let $\mathcal{O}=\mathbb{Z}[\chi]$. Moreover, we let $\mathfrak{P}$ be a maximal ideal of $\mathcal{O}$ above $p$ with residue field $\mathbb{F}$ and $\widehat{\mathcal{O}}$ be the completion of $\mathcal{O}$ at $\mathfrak{P}$. Furthermore, let $K=\mathbb{Q}_{p}(\chi)$ be the field of fractions of $\widehat{\mathcal{O}}$ and $\bar{\chi}$ be $\chi$ followed by the natural projection $\mathcal{O} \rightarrow \mathbb{F}$.

Denote by $M_{k}(N, \chi ; \mathcal{O})$ the sub- $\mathcal{O}$-module generated by those modular forms whose (standard) $q$-expansion has coefficients in $\mathcal{O}$. It follows from the $q$-expansion principle that

$$
M_{k}(N, \chi ; \mathcal{O}) \cong \operatorname{Hom}_{\mathcal{O}}\left(\mathbb{T}_{\mathcal{O}}\left(M_{k}(N, \chi)\right), \mathcal{O}\right)
$$

and that hence $M_{k}(N, \chi ; \mathcal{O}) \otimes_{\mathcal{O}} \mathbb{C} \cong M_{k}(N, \chi)$. We put

$$
M_{k}(N, \bar{\chi} ; \mathbb{F}):=M_{k}(N, \chi ; \mathcal{O}) \otimes_{\mathcal{O}} \mathbb{F} \cong \operatorname{Hom}_{\mathbb{F}}\left(\mathbb{T}_{\mathcal{O}}\left(M_{k}(N, \chi)\right), \mathbb{F}\right)
$$

and call the elements of this space mod $p$ modular forms. The Hecke algebra $\mathbb{T}_{\mathcal{O}}\left(M_{k}(N, \chi)\right)$ acts naturally and it follows that $\mathbb{T}_{\mathcal{O} \rightarrow \mathbb{F}}\left(M_{k}(N, \chi)\right) \cong \mathbb{T}_{\mathbb{F}}\left(M_{k}(N, \chi ; \mathbb{F})\right)$. Similar statements hold for the cuspidal and the Eisenstein subspaces and we use similar notations.

We call a maximal ideal $\mathfrak{m}$ of $\mathbb{T}_{\mathcal{O} \rightarrow \mathbb{F}}\left(M_{k}(N, \chi ; \mathcal{O})\right.$ (respectively, the corresponding maximal ideal of $\left.\mathbb{T}_{\mathcal{O} \rightarrow \widehat{\mathcal{O}}}\left(M_{k}(N, \chi ; \mathcal{O})\right)\right)$ non-Eisenstein if and only if

$$
S_{k}(N, \bar{\chi} ; \mathbb{F})_{\mathfrak{m}} \cong M_{k}(N, \bar{\chi} ; \mathbb{F})_{\mathfrak{m}}
$$

Otherwise, we call $\mathfrak{m}$ Eisenstein.

We now include a short discussion of minimal and maximal primes, in view of Proposition 3.5 Write $\mathbb{T}_{\widehat{\mathcal{O}}}$ for $\mathbb{T}_{\mathcal{O} \rightarrow \widehat{\mathcal{O}}}\left(S_{k}(N, \chi)\right)$. Let $\mathfrak{m}$ be a maximal ideal of $\mathbb{T}_{\widehat{\mathcal{O}}}$. It corresponds to a Gal $\left(\overline{\mathbb{F}}_{p} / \mathbb{F}\right)$ conjugacy class of normalised eigenforms in $S_{k}(N, \bar{\chi} ; \mathbb{F})$. That means for each $n \in \mathbb{N}$ that the minimal polynomial of $T_{n}$ acting on $S_{k}(N, \bar{\chi} ; \mathbb{F})_{\mathfrak{m}}$ is equal to a power of the minimal polynomial of the coefficient $a_{n}$ of each member of the conjugacy class. Moreover, $\mathfrak{p}$ corresponds precisely as above to a $\operatorname{Gal}\left(\overline{\mathbb{Q}}_{p} / K\right)$-conjugacy class of normalised eigenforms in $S_{k}(N, \chi ; \mathcal{O}) \otimes_{\mathcal{O}} K$.

Suppose that $\mathfrak{m}$ contains minimal primes $\mathfrak{p}_{i}$ for $i=1, \ldots, r$. Then the normalised eigenforms corresponding to the $\mathfrak{p}_{i}$ are congruent to one another modulo a prime above $p$. Conversely, any congruence arises in this way. Thus, a maximal ideal $\mathfrak{m}$ of $\mathbb{T}_{\widehat{\mathcal{O}}}$ is Eisenstein if and only if it contains a minimal prime corresponding to a conjugacy class of Eisenstein series. As it is the reduction of a reducible representation, the mod $p$ Galois representation corresponding to a non-Eisenstein prime is reducible. It should be possible to show the converse.

\section{Modular symbols}

We now recall the modular symbols formalism and prove two useful results on base change and torsion. The main references for the definitions are [19] and [21]. 
Let $R$ be a ring, $\Gamma \leq \mathrm{SL}_{2}(\mathbb{Z})$ a subgroup and $V$ a left $R[\Gamma]$-module. Recall that $\mathbb{P}^{1}(\mathbb{Q})=\mathbb{Q} \cup\{\infty\}$ is the set of cusps of $\mathrm{SL}_{2}(\mathbb{Z})$, which carries a natural $\mathrm{SL}_{2}(\mathbb{Z})$-action via fractional linear transformations. We define the $R$-modules

$$
\mathcal{M}_{R}:=R\left[\{\alpha, \beta\} \mid \alpha, \beta \in \mathbb{P}^{1}(\mathbb{Q})\right] /\left\langle\{\alpha, \alpha\},\{\alpha, \beta\}+\{\beta, \gamma\}+\{\gamma, \alpha\} \mid \alpha, \beta, \gamma \in \mathbb{P}^{1}(\mathbb{Q})\right\rangle
$$

and $\mathcal{B}_{R}:=R\left[\mathbb{P}^{1}(\mathbb{Q})\right]$. They are connected via the boundary map $\delta: \mathcal{M}_{R} \rightarrow \mathcal{B}_{R}$ which is given by $\{\alpha, \beta\} \mapsto \beta-\alpha$. Both are equipped with the natural left $\Gamma$-actions. Also let $\mathcal{M}_{R}(V):=\mathcal{M}_{R} \otimes_{R} V$ and $\mathcal{B}_{R}(V):=\mathcal{B}_{R} \otimes_{R} V$ with the left diagonal $\Gamma$-action. We call the $\Gamma$-coinvariants

$$
\mathcal{M}_{R}(\Gamma, V):=\mathcal{M}_{R}(V)_{\Gamma}=\mathcal{M}_{R}(V) /\left\langle(x-g x) \mid g \in \Gamma, x \in \mathcal{M}_{R}(V)\right\rangle
$$

the space of $(\Gamma, V)$-modular symbols. Furthermore, the space of $(\Gamma, V)$-boundary symbols is defined as the $\Gamma$-coinvariants

$$
\mathcal{B}_{R}(\Gamma, V):=\mathcal{B}_{R}(V)_{\Gamma}=\mathcal{B}_{R}(V) /\left\langle(x-g x) \mid g \in \Gamma, x \in \mathcal{B}_{R}(V)\right\rangle
$$

The boundary map $\delta$ induces the boundary map $\mathcal{M}_{R}(\Gamma, V) \rightarrow \mathcal{B}_{R}(\Gamma, V)$. Its kernel is denoted by $\mathcal{C M}_{R}(\Gamma, V)$ and is called the space of cuspidal $(\Gamma, V)$-modular symbols.

Let now $N \geq 1$ and $k \geq 2$ be integers and $\chi:(\mathbb{Z} / N \mathbb{Z})^{\times} \rightarrow R^{\times}$be a character, i.e. a group homomorphism, such that $\chi(-1)=(-1)^{k}$ in $R$. Write $V_{k-2}(R)$ for the homogeneous polynomials of degree $k-2$ over $R$ in two variables, equipped with the natural $\Gamma_{0}(N)$-action. Denote by $V_{k-2}^{\chi}(R)$ the tensor product $V_{k-2}(R) \otimes_{R} R^{\chi}$ for the diagonal $\Gamma_{0}(N)$-action which on $R^{\chi}$ comes from the isomorphism $\Gamma_{0}(N) / \Gamma_{1}(N) \cong(\mathbb{Z} / N \mathbb{Z})^{\times}$given by sending $\left(\begin{array}{ll}a & b \\ c & d\end{array}\right)$ to $d$ followed by $\chi^{-1}$.

We use the notation $\mathcal{M}_{k}(N, \chi ; R)$ for $\mathcal{M}\left(\Gamma_{0}(N), V_{k-2}^{\chi}(R)\right)$, as well as similarly for the boundary and the cuspidal spaces. The natural action of the matrix $\eta=\left(\begin{array}{cc}-1 & 0 \\ 0 & 1\end{array}\right)$ gives an involution on all of these spaces. We will denote by the superscript ${ }^{+}$the subspace invariant under this involution, and by the superscript ${ }^{-}$the anti-invariant one. On all modules discussed so far one has Hecke operators $T_{n}$ for all $n \in \mathbb{N}$ and diamond operators. For a definition see [19].

Lemma 3.1 Let $R, \Gamma$ and $V$ as above and let $R \rightarrow S$ be a ring homomorphism. Then

$$
\mathcal{M}(\Gamma, V) \otimes_{R} S \cong \mathcal{M}\left(\Gamma, V \otimes_{R} S\right)
$$

Proof. This follows immediately from the fact that tensoring and taking coinvariants are both right exact.

Proposition 3.2 Let $R$ be a local integral domain of characteristic zero with principal maximal ideal $\mathfrak{m}=(\pi)$ and residue field $\mathbb{F}$ of characteristic $p$. Also let $N \geq 1, k \geq 2$ be integers and $\chi:(\mathbb{Z} / N \mathbb{Z})^{\times} \rightarrow R^{\times}$a character such that $\chi(-1)=(-1)^{k}$. Suppose (i) that $p \geq 5$ or (ii) that $p=2$ and $N$ is divisible by a prime which is 3 modulo 4 or by 4 or (iii) that $p=3$ and $N$ is divisible by a prime which is 2 modulo 3 or by 9 . Then the following statements hold: 
(a) If $k \geq 3$, then $\mathcal{M}_{k}(N, \chi ; R)[\pi]=\left(V_{k-2}^{\chi}(\mathbb{F})\right)^{\Gamma_{0}(N)}$.

(b) If $k=2$ or if $3 \leq k \leq p+2$ and $p \nmid N$, then $\mathcal{M}_{k}(N, \chi ; R)[\pi]=0$.

Proof. The conditions assure that the group $\Gamma_{0}(N)$ does not have any stabiliser of order $2 p$ for its action on the upper half plane. Hence, by [21], Theorem 6.1, the modular symbols space $\mathcal{M}_{k}(N, \chi ; R)$ is isomorphic to $H^{1}\left(\Gamma_{0}(N), V_{k-2}^{\chi}(R)\right)$. The arguments are now precisely those of the beginning of the proof of [22], Proposition 2.6.

\section{Hecke algebras of modular symbols and the Eichler-Shimura isomorphism}

From Lemma 3.1 one deduces a natural surjection

$$
\mathbb{T}_{\mathcal{O} \rightarrow \mathbb{F}}\left(\mathcal{M}_{k}(N, \chi ; \mathcal{O})\right) \rightarrow \mathbb{T}_{\mathbb{F}}\left(\mathcal{M}_{k}(N, \bar{\chi} ; \mathbb{F})\right) .
$$

In the same way, one also obtains

$$
\mathbb{T}_{\mathcal{O}}\left(\mathcal{M}_{k}(N, \chi ; \mathcal{O})\right) \rightarrow \mathbb{T}_{\mathcal{O}}\left(\mathcal{M}_{k}(N, \chi ; \mathcal{O}) / \text { torsion }\right) \cong \mathbb{T}_{\mathcal{O}}\left(\mathcal{M}_{k}(N, \chi ; \mathbb{C})\right),
$$

where one uses for the isomorphism that the Hecke operators are already defined over $\mathcal{O}$. A similar statement holds for the cuspidal subspace.

We call a maximal prime $\mathfrak{m}$ of $\mathbb{T}_{\mathcal{O} \rightarrow \widehat{\mathcal{O}}}\left(\mathcal{M}_{k}(N, \chi ; \mathcal{O})\right)$ (respectively the corresponding prime of $\left.\mathbb{T}_{\mathcal{O} \rightarrow \mathbb{F}}\left(\mathcal{M}_{k}(N, \chi ; \mathcal{O})\right)\right)$ non-torsion if

$$
\mathcal{M}_{k}(N, \chi ; \widehat{\mathcal{O}})_{\mathfrak{m}} \cong\left(\mathcal{M}_{k}(N, \chi ; \widehat{\mathcal{O}}) / \text { torsion }\right)_{\mathfrak{m}}
$$

This is equivalent to the height of $\mathfrak{m}$ being 1 . Proposition 3.2 tells us some cases in which all primes are non-torsion.

Theorem 3.3 (Eichler-Shimura) There are isomorphisms respecting the Hecke operators

(a) $M_{k}(N, \chi) \oplus S_{k}(N, \chi)^{\vee} \cong \mathcal{M}_{k}(N, \chi ; \mathbb{C})$,

(b) $S_{k}(N, \chi) \oplus S_{k}(N, \chi)^{\vee} \cong \mathcal{C M}_{k}(N, \chi ; \mathbb{C})$,

(c) $S_{k}(N, \chi) \cong \mathcal{C M}_{k}(N, \chi ; \mathbb{C})^{+}$.

Proof. Parts (a) and (b) are [6], Theorem 12.2.2, together with the comparison of [21], Theorem 6.1. We use that the space of anti-holomorphic cusp forms is dual to the space of holomorphic cusp forms. Part (c) is a direct consequence of (b).

Corollary 3.4 There are isomorphisms

$$
\mathbb{T}_{\mathcal{O}}\left(S_{k}(N, \chi)\right) \cong \mathbb{T}_{\mathcal{O}}\left(\mathcal{C} \mathcal{M}_{k}(N, \chi ; \mathbb{C})\right) \cong \mathbb{T}_{\mathcal{O}}\left(\mathcal{C} \mathcal{M}_{k}(N, \chi ; \mathbb{C})^{+}\right)
$$

given by sending $T_{n}$ to $T_{n}$ for all positive $n$. 


\subsection{The stop criterion}

Although it is impossible to determine a priori the dimension of the local factor of the Hecke algebra associated with a given modular form mod $p$, Corollary 3.8 implies that the computation of Hecke operators can be stopped when the algebra generated has reached a certain dimension that is computed along the way. This criterion has turned out to be extremely useful and has made possible some of our computations which would not have been feasible using the Hecke bound naively. See Section 4 for a short discussion of this issue.

\section{Some commutative algebra}

We collect some useful statements from commutative algebra, which will be applied to Hecke algebras in the sequel.

Proposition 3.5 Let $R$ be an integral domain of characteristic zero which is a finitely generated $\mathbb{Z}$ module. Write $\widehat{R}$ for the completion of $R$ at a maximal ideal of $R$ and denote by $\mathbb{F}$ the residue field and by $K$ the fraction field of $\widehat{R}$. Let furthermore $A$ be a commutative R-algebra which is finitely generated as an $R$-module. For any ring homomorphism $R \rightarrow S$ write $A_{S}$ for $A \otimes_{R} S$. Then the following statements hold.

(a) The Krull dimension of $A_{\widehat{R}}$ is less than or equal to 1 . The maximal ideals of $A_{\widehat{R}}$ correspond bijectively under taking pre-images to the maximal ideals of $A_{\mathbb{F}}$. Primes $\mathfrak{p}$ of height 0 which are contained in a prime of height 1 of $A_{\widehat{R}}$ are in bijection with primes of $A_{K}$ under extension (i.e. $\left.\mathfrak{p} A_{K}\right)$, for which the notation $\mathfrak{p}^{e}$ will be used.

Under these correspondences, one has $A_{\mathbb{F}, \mathfrak{m}} \cong A_{\widehat{R}, \mathfrak{m}} \otimes_{\widehat{R}} \mathbb{F}$ and $A_{K, \mathfrak{p}^{e}} \cong A_{\widehat{R}, \mathfrak{p}}$.

(b) The algebra $A_{\widehat{R}}$ decomposes as

$$
A_{\widehat{R}} \cong \prod_{\mathfrak{m}} A_{\widehat{R}, \mathfrak{m}}
$$

where the product runs over the maximal ideals $\mathfrak{m}$ of $A_{\widehat{R}}$.

(c) The algebra $A_{\mathbb{F}}$ decomposes as

$$
A_{\mathbb{F}} \cong \prod_{\mathfrak{m}} A_{\mathbb{F}, \mathfrak{m}}
$$

where the product runs over the maximal ideals $\mathfrak{m}$ of $A_{\mathbb{F}}$.

(d) The algebra $A_{K}$ decomposes as

$$
A_{K} \cong \prod_{\mathfrak{p}} A_{K, \mathfrak{p} e} \cong \prod_{\mathfrak{p}} A_{\widehat{R}, \mathfrak{p}}
$$

where the products run over the minimal prime ideals $\mathfrak{p}$ of $A_{\widehat{R}}$ which are contained in a prime ideal of height 1 . 
Proof. As $A_{\widehat{R}}$ is a finitely generated $\widehat{R}$-module, $A_{\widehat{R}} / \mathfrak{p}$ with a prime $\mathfrak{p}$ is an integral domain which is a finitely generated $\widehat{R}$-module. Hence, it is either a finite field or a finite extension of $\widehat{R}$. This proves that the height of $\mathfrak{p}$ is less than or equal to 1 . The correspondences and the isomorphisms of Part (a) are easily verified. The decompositions in Parts (b) and (c) are [10], Corollary 7.6. Part (d) follows by tensoring (b) over $\widehat{R}$ with $K$.

Similar decompositions for $A$-modules are derived by applying the idempotents of the decompositions of Part (b).

Proposition 3.6 Assume the set-up of Proposition 3.5 and let $M, N$ be A-modules which as $R$ modules are free of finite rank. Suppose that

(a) $M \otimes_{R} \mathbb{C} \cong N \otimes_{R} \mathbb{C}$ as $A \otimes_{R} \mathbb{C}$-modules, or

(b) $M \otimes_{R} \bar{K} \cong N \otimes_{R} \bar{K}$ as $A \otimes_{R} \bar{K}$-modules.

Then for all prime ideals $\mathfrak{m}$ of $A_{\mathbb{F}}$ corresponding to height 1 primes of $A_{\widehat{R}}$ the equality

$$
\operatorname{dim}_{\mathbb{F}}\left(M \otimes_{R} \mathbb{F}\right)_{\mathfrak{m}}=\operatorname{dim}_{\mathbb{F}}\left(N \otimes_{R} \mathbb{F}\right)_{\mathfrak{m}}
$$

holds.

Proof. As for $A$, we also write $M_{K}$ for $M \otimes_{R} K$ and similarly for $N$ and $\widehat{R}, \mathbb{F}$, etc. By choosing an isomorphism $\mathbb{C} \cong \bar{K}$, it suffices to prove Part (b). Using Proposition 3.5. Part (d), the isomorphism $M \otimes_{R} \bar{K} \cong N \otimes_{R} \bar{K}$ can be rewritten as

$$
\bigoplus_{\mathfrak{p}}\left(M_{K, \mathfrak{p}^{e}} \otimes_{K} \bar{K}\right) \cong \bigoplus_{\mathfrak{p}}\left(N_{K, \mathfrak{p}^{e}} \otimes_{K} \bar{K}\right)
$$

where the sums run over the minimal primes $\mathfrak{p}$ of $A_{\widehat{R}}$ which are properly contained in a maximal prime. Hence, an isomorphism $M_{K, \mathfrak{p}^{e}} \otimes_{K} \bar{K} \cong N_{K, \mathfrak{p}^{e}} \otimes_{K} \bar{K}$ exists for each $\mathfrak{p}$. Since for each maximal ideal $\mathfrak{m}$ of $A_{\widehat{R}}$ of height 1 we have by Proposition 3.5

$$
M_{\widehat{R}, \mathfrak{m}} \otimes_{\widehat{R}} K \cong \bigoplus_{\mathfrak{p} \subseteq \mathfrak{m} \min .} M_{K, \mathfrak{p} e}
$$

and similarly for $N$, we get

$$
\begin{aligned}
\operatorname{dim}_{\mathbb{F}} M_{\mathbb{F}, \mathfrak{m}} & =\mathrm{rk}_{\widehat{R}} M_{\widehat{R}, \mathfrak{m}}=\sum_{\mathfrak{p} \subseteq \mathfrak{m} \min .} \operatorname{dim}_{K} M_{K, \mathfrak{p}^{e}} \\
& =\sum_{\mathfrak{p} \subseteq \mathfrak{m} \min .} \operatorname{dim}_{K} N_{K, \mathfrak{p}^{e}}=\operatorname{rk}_{\widehat{R}} N_{\widehat{R}, \mathfrak{m}}=\operatorname{dim}_{\mathbb{F}} N_{\mathbb{F}, \mathfrak{m}} .
\end{aligned}
$$

This proves the proposition. 


\section{The stop criterion}

Proposition 3.7 Let $\mathfrak{m}$ be a maximal ideal of $\mathbb{T}_{\mathcal{O} \rightarrow \mathbb{F}}\left(\mathcal{M}_{k}(N, \chi ; \mathcal{O})\right)$ which is non-torsion and nonEisenstein. Then the following statements hold:

(a) $\mathcal{C M}_{k}(N, \bar{\chi} ; \mathbb{F})_{\mathfrak{m}} \cong \mathcal{M}_{k}(N, \bar{\chi} ; \mathbb{F})_{\mathfrak{m}}$.

(b) $2 \cdot \operatorname{dim}_{\mathbb{F}} S_{k}(N, \bar{\chi} ; \mathbb{F})_{\mathfrak{m}}=\operatorname{dim}_{\mathbb{F}} \mathcal{C} \mathcal{M}_{k}(N, \bar{\chi} ; \mathbb{F})_{\mathfrak{m}}$

(c) If $p \neq 2$, then $\operatorname{dim}_{\mathbb{F}} S_{k}(N, \bar{\chi} ; \mathbb{F})_{\mathfrak{m}}=\operatorname{dim}_{\mathbb{F}} \mathcal{C} \mathcal{M}_{k}(N, \bar{\chi} ; \mathbb{F})_{\mathfrak{m}}^{+}$.

Proof. Part (c) follows directly from Part (b) by decomposing $\mathcal{C M}_{k}(N, \bar{\chi} ; \mathbb{F})$ into a direct sum of its plus- and its minus-part. Statements (a) and (b) will be concluded from Proposition 3.6 More precisely, it allows us to derive from Theorem 3.3 that

$$
\begin{aligned}
& \operatorname{dim}_{\mathbb{F}}\left(\left(\mathcal{M}_{k}(N, \chi ; \mathcal{O}) / \text { torsion }\right) \otimes_{\mathcal{O}} \mathbb{F}\right)_{\mathfrak{m}} \\
= & \operatorname{dim}_{\mathbb{F}}\left(\operatorname{Eis}_{k}(N, \bar{\chi} ; \mathbb{F}) \oplus S_{k}(N, \bar{\chi} ; \mathbb{F}) \oplus S_{k}(N, \bar{\chi} ; \mathbb{F})^{\vee}\right)_{\mathfrak{m}}
\end{aligned}
$$

and

$$
\operatorname{dim}_{\mathbb{F}}\left(\left(\mathcal{C} \mathcal{M}_{k}(N, \chi ; \mathcal{O}) / \text { torsion }\right) \otimes_{\mathcal{O}} \mathbb{F}\right)_{\mathfrak{m}}=2 \cdot \operatorname{dim}_{\mathbb{F}}\left(S_{k}(N, \bar{\chi} ; \mathbb{F})\right)_{\mathfrak{m}}
$$

The latter proves Part (b), since $\mathfrak{m}$ is non-torsion. As by the definition of a non-Eisenstein prime $\operatorname{Eis}_{k}(N, \bar{\chi} ; \mathbb{F})_{\mathfrak{m}}=0$ and again since $\mathfrak{m}$ is non-torsion, it follows that

$$
\operatorname{dim}_{\mathbb{F}} \mathcal{C} \mathcal{M}_{k}(N, \bar{\chi} ; \mathbb{F})_{\mathfrak{m}}=\operatorname{dim}_{\mathbb{F}} \mathcal{M}_{k}(N, \bar{\chi} ; \mathbb{F})_{\mathfrak{m}}
$$

which implies Part (a).

We will henceforth often regard non-Eisenstein non-torsion primes as in the proposition as maximal primes of $\mathbb{T}_{\mathbb{F}}\left(S_{k}(N, \bar{\chi} ; \mathbb{F})\right)=\mathbb{T}_{\mathcal{O} \rightarrow \mathbb{F}}\left(S_{k}(N, \chi)\right)$.

Corollary 3.8 (Stop Criterion) Let $\mathfrak{m}$ be a maximal ideal of $\mathbb{T}_{\mathbb{F}}\left(S_{k}(N, \bar{\chi} ; \mathbb{F})\right)$ which is non-Eisenstein and non-torsion.

(a) One has $\operatorname{dim}_{\mathbb{F}} \mathcal{M}_{k}(N, \bar{\chi} ; \mathbb{F})_{\mathfrak{m}}=2 \cdot \operatorname{dim}_{\mathbb{F}} \mathbb{T}_{\mathbb{F}}\left(\mathcal{M}_{k}(N, \bar{\chi} ; \mathbb{F})\right)_{\mathfrak{m}}$ if and only if

$$
\mathbb{T}_{\mathbb{F}}\left(S_{k}(N, \bar{\chi} ; \mathbb{F})\right)_{\mathfrak{m}} \cong \mathbb{T}_{\mathbb{F}}\left(\mathcal{C} \mathcal{M}_{k}(N, \bar{\chi} ; \mathbb{F})\right)_{\mathfrak{m}}
$$

(b) One has $\operatorname{dim}_{\mathbb{F}} \mathcal{C} \mathcal{M}_{k}(N, \bar{\chi} ; \mathbb{F})_{\mathfrak{m}}=2 \cdot \operatorname{dim}_{\mathbb{F}} \mathbb{T}_{\mathbb{F}}\left(\mathcal{C} \mathcal{M}_{k}(N, \bar{\chi} ; \mathbb{F})\right)_{\mathfrak{m}}$ if and only if

$$
\mathbb{T}_{\mathbb{F}}\left(S_{k}(N, \bar{\chi} ; \mathbb{F})\right)_{\mathfrak{m}} \cong \mathbb{T}_{\mathbb{F}}\left(\mathcal{C} \mathcal{M}_{k}(N, \bar{\chi} ; \mathbb{F})\right)_{\mathfrak{m}}
$$

(c) Assume $p \neq 2$. One has $\operatorname{dim}_{\mathbb{F}} \mathcal{C M}_{k}(N, \bar{\chi} ; \mathbb{F})_{\mathfrak{m}}^{+}=\operatorname{dim}_{\mathbb{F}} \mathbb{T}_{\mathbb{F}}\left(\mathcal{C M}_{k}(N, \bar{\chi} ; \mathbb{F})\right)_{\mathfrak{m}}$ if and only if

$$
\mathbb{T}_{\mathbb{F}}\left(S_{k}(N, \bar{\chi} ; \mathbb{F})\right)_{\mathfrak{m}} \cong \mathbb{T}_{\mathbb{F}}\left(\mathcal{C} \mathcal{M}_{k}(N, \bar{\chi} ; \mathbb{F})^{+}\right)_{\mathfrak{m}}
$$


Proof. We only prove (a), as (b) and (c) are similar. From Part (b) of Proposition 3.7 and the fact that the $\mathbb{F}$-dimension of the algebra $\mathbb{T}_{\mathbb{F}}\left(S_{k}(N, \bar{\chi} ; \mathbb{F})\right)_{\mathfrak{m}}$ is equal to the one of $S_{k}(N, \bar{\chi} ; \mathbb{F})$, as they are dual to each other, it follows that

$$
2 \cdot \operatorname{dim}_{\mathbb{F}} \mathbb{T}_{\mathbb{F}}\left(S_{k}(N, \bar{\chi} ; \mathbb{F})\right)_{\mathfrak{m}}=\operatorname{dim}_{\mathbb{F}}\left(\mathcal{C} \mathcal{M}_{k}(N, \bar{\chi} ; \mathbb{F})\right)_{\mathfrak{m}}
$$

The result is now a direct consequence of Equations 3.1 and 3.2 and Corollary 3.4

Note that the first line of each statement only uses modular symbols and not modular forms, but it allows us to make statements involving modular forms. This is the aforementioned stop criterion; the computation of Hecke operators can be stopped if this equality is reached.

We now list some results concerning the validity of the equivalent statements of Corollary 3.8 The reader can also consult [12] for general results in the ordinary and distinguished case.

Proposition 3.9 Let $p \geq 5$ be a prime, $k \geq 2$ and $N \geq 5$ with $p \nmid N$ integers, $\mathbb{F}$ a finite extension of $\mathbb{F}_{p}, \bar{\chi}:(\mathbb{Z} / N \mathbb{Z})^{\times} \rightarrow \mathbb{F}^{\times}$a character and $\mathfrak{m}$ a maximal ideal of $\mathbb{T}_{\mathbb{F}}\left(S_{k}(N, \bar{\chi} ; \mathbb{F})\right)$ which is nonEisenstein and non-torsion. Suppose (i) that $2 \leq k \leq p-1$ or (ii) that $k \in\{p, p+1\}$ and $\mathfrak{m}$ is ordinary. Then

$$
\mathbb{T}_{\mathbb{F}}\left(S_{k}(N, \bar{\chi} ; \mathbb{F})\right)_{\mathfrak{m}} \cong \mathbb{T}_{\mathbb{F}}\left(\mathcal{C} \mathcal{M}_{k}(N, \bar{\chi} ; \mathbb{F})\right)_{\mathfrak{m}} \cong \mathbb{T}_{\mathbb{F}}\left(\mathcal{C} \mathcal{M}_{k}(N, \bar{\chi} ; \mathbb{F})^{+}\right)_{\mathfrak{m}}
$$

Proof. Using the comparison with group cohomology of [21], Theorem 6.1, the result follows under Assumption (i) from [9], Theorem 5.2, and is proved under Assumption (ii) in [22], Corollary 6.9, for the case of the group $\Gamma_{1}(N)$ and no Dirichlet character. The passage to a character is established by [22], Theorem 7.4 and the remark following it. One identifies the mod $p$ modular forms appearing with corresponding Katz forms using Carayol's Lemma ([8], Prop. 1.10).

We end this section by stating the so-called Sturm bound (also called the Hecke bound), which gives the best a priori upper bound for how many Hecke operators are needed to generate all the Hecke algebra. We only need it in our algorithm in cases in which it is theoretically not known that the stop criterion will be reached. This will enable the algorithm to detect if the Hecke algebra on modular symbols is not isomorphic to the corresponding one on cuspidal modular forms.

Proposition 3.10 (Sturm bound) The Hecke algebra $\mathbb{T}_{\mathbb{C}}\left(S_{k}(N, \chi)\right)$ can be generated as an algebra by the Hecke operators $T_{l}$ for all primes $l$ smaller than or equal to $\frac{k N}{12} \prod_{q \mid N, q \text { prime }}\left(1+\frac{1}{q}\right)$.

Proof. This is discussed in detail in Chapter 11 of [19].

\subsection{Algorithm}

In this section we present a sketch of the algorithm that we used for our computations. The MAGMA code can be downloaded from the second author's webpage and a manual is included as Appendix A Input: Integers $N \geq 1, k \geq 2$, a finite field $\mathbb{F}$, a character $\chi:(\mathbb{Z} / N \mathbb{Z})^{\times} \rightarrow \mathbb{F}^{\times}$and for each prime $l$ less than or equal to the Sturm bound an irreducible polynomial $f_{l} \in \mathbb{F}[X]$.

Output: An $\mathbb{F}$-algebra. 
- $M \leftarrow \mathcal{C} \mathcal{M}_{k}(N, \chi ; \mathbb{F}), l \leftarrow 1, L \leftarrow$ empty list.

- repeat

$-l \leftarrow$ next prime after $l$.

- Compute $T_{l}$ on $M$ and append it to the list $L$.

- $M \leftarrow$ the restriction of $M$ to the $f_{l}$-primary subspace for $T_{l}$, i.e. to the biggest subspace of $M$ on which the minimal polynomial of $T_{l}$ is a power of $f_{l}$.

- $A \leftarrow$ the $\mathbb{F}$-algebra generated by the restrictions to $M$ of $T_{2}, T_{3}, \ldots, T_{l}$.

- until $2 \cdot \operatorname{dim}(A)=\operatorname{dim}(M)$ [the stop criterion] or $l>$ Sturm bound.

- return $A$.

The $f_{l}$ should, of course, be chosen as the minimal polynomials of the coefficients $a_{l}(f)$ of the normalised eigenform $f \in S_{k}(N, \chi ; \overline{\mathbb{F}})$ whose local Hecke algebra one wants to compute. Suppose the algorithm stops at the prime $q$. If $q$ is bigger than the Sturm bound, the equivalent conditions of Corollary 3.8 do not hold. In that case the output should be disregarded. Otherwise, $A$ is isomorphic to a direct product of the form $\prod_{\mathfrak{m}} \mathbb{T}\left(S_{k}(N, \chi ; \mathbb{F})\right)_{\mathfrak{m}}$ where the $\mathfrak{m}$ are those maximal ideals such that the minimal polynomials of $T_{2}, T_{3}, \ldots, T_{q}$ on $\mathbb{T}\left(S_{k}(N, \chi ; \mathbb{F})\right)_{\mathfrak{m}}$ are equal to powers of $f_{2}, f_{3}, \ldots, f_{q}$. It can happen that $A$ consists of more than one factor. Hence, one should still decompose $A$ into its local factors. Alternatively, one can also replace the last line but one in the algorithm by

- until $((2 \cdot \operatorname{dim}(A)=\operatorname{dim}(M))$ and $A$ is local $)$ or $l>$ Sturm bound,

which ensures that the output is a local algebra. In practice, one modifies the algorithm such that not for every prime $l$ a polynomial $f_{l}$ need be given, but that the algorithm takes each irreducible factor of the minimal polynomial of $T_{l}$ if no $f_{l}$ is known. It is also useful to choose the order how $l$ runs through the primes. For example, one might want to take $l=p$ at an early stage with $p$ the characteristic of $\mathbb{F}$, if one knows that this operator is needed, which is the case in all computations concerning Question 1.9

\section{Computational results}

In view of Question 1.9, we produced 384 examples of odd irreducible continuous Galois representations $\operatorname{Gal}(\overline{\mathbb{Q}} / \mathbb{Q}) \rightarrow \mathrm{GL}_{2}\left(\overline{\mathbb{F}}_{p}\right)$ that are completely split at $p$. The results are documented in the accompanying tables (Appendix B). The complete data can be downloaded from the second author's webpage.

The Galois representations were created either by class field theory or from an irreducible integer polynomial whose Galois group embeds into $\mathrm{GL}_{2}\left(\overline{\mathbb{F}}_{p}\right)$. All examples but one are dihedral; the remaining one is icosahedral. For each of these an eigenform was computed giving rise to it. The Gorenstein defect of the corresponding local Hecke algebra factor turned out always to be 2, supporting Question 1.9 
The authors preferred to proceed like this, instead of computing all Hecke algebras mod $p$ in weight $p$ for all "small" primes $p$ and all "small" levels, since non-dihedral examples in which the assumptions of Question 1.9 are satisfied are very rare.

\subsection{Table entries}

For every computed local Hecke algebra enough data are stored to recreate it as an abstract algebra and important characteristics are listed in the tables of Appendix B. A sample table entry is the following.

\begin{tabular}{||c|c|c|c|c|c|c|c|c|c||}
\hline Level & Wt & ResD & Dim & EmbDim & NilO & GorDef & \#Ops & $\#(\mathrm{p}<\mathrm{HB})$ & Gp \\
\hline 5939 & 5 & 3 & 12 & 3 & 5 & 2 & 5 & 366 & $D_{7}$ \\
\hline
\end{tabular}

Each entry corresponds to the Galois conjugacy class of an eigenform $f$ mod $p$ with associated local Hecke algebra $A$. The first and the second column indicate the level and the weight of $f$. The latter is in all examples equal to the characteristic of the base field $k$ (a finite extention of $\mathbb{F}_{p}$ ) of the algebra. Let $\mathfrak{m}_{A}$ denote the maximal ideal of $A$. Then ResD stands for the degree of $K=A / \mathfrak{m}_{A}$ over $\mathbb{F}_{p}$. Let us consider $A \otimes_{k} K$. It decomposes into a direct product of a local $K$-algebra $B$ and its $\operatorname{Gal}(K / k)$-conjugates. The $K$-dimension of $B$ (which is equal to the $k$-dimension of $A$ ) is recorded in the fourth column.

Let $\mathfrak{m}_{B}$ be the maximal ideal of $B$. The embedding dimension EmbDim is the $K$-dimension of $\mathfrak{m}_{B} / \mathfrak{m}_{B}^{2}$. By Nakayama's Lemma this is the minimal number of $B$-generators for $\mathfrak{m}_{B}$. The nilpotency order NilO is the maximal integer $n$ such that $\mathfrak{m}_{B}^{n}$ is not the zero ideal. The column GorDef contains the Gorenstein defect of $B$ (which is the same as the Gorenstein defect of $A$ ).

By \#Ops it is indicated how many Hecke operators were used to generate the algebra $A$, applying the stop criterion (Corollary 3.8). This is contrasted with the number of primes smaller than the Sturm bound (Proposition 3.10, it is also called the Hecke bound), denoted by $\#(\mathrm{p}<\mathrm{HB})$. One immediately observes that the stop criterion is very efficient. Whereas the Sturm bound is roughly linear in the level, in 365 of the 384 calculated examples, less than 10 Hecke operators sufficed, in 252 examples even 5 were enough.

The final column contains the image of the $\bmod p$ Galois representation attached to $f$ as an abstract group.

\subsection{Dihedral examples}

All Hecke algebras except one in our tables correspond to eigenforms whose Galois representations are dihedral, since these are by far the easiest to obtain explicitly as one can use class field theory. This is explained now.

Let $p$ be a prime and $d$ a square-free integer which is $1 \bmod 4$ and not divisible by $p$. We denote by $K$ the quadratic field $\mathbb{Q}(\sqrt{d})$. Further we consider an unramified character $\chi: \operatorname{Gal}(\overline{\mathbb{Q}} / K) \rightarrow \overline{\mathbb{F}}_{p}{ }^{\times}$ of order $n \geq 3$. We assume that its inverse $\chi^{-1}$ is equal to $\chi$ conjugated by $\sigma$, denoted $\chi^{\sigma}$, for $\sigma$ (a 
lift of) the non-trivial element of $\operatorname{Gal}(K / \mathbb{Q})$. The induced representation

$$
\rho_{\chi}:=\operatorname{Ind}_{\operatorname{Gal}(\overline{\mathbb{Q}} / K)}^{\operatorname{Gal}(\overline{\mathbb{Q}} / \mathbb{Q})}(\chi): \operatorname{Gal}(\overline{\mathbb{Q}} / \mathbb{Q}) \rightarrow \mathrm{GL}_{2}\left(\overline{\mathbb{F}}_{p}\right)
$$

is irreducible and its image is the dihedral group $D_{n}$ of order $2 n$. If $l$ is a prime not dividing $2 d$, we have $\rho_{\chi}\left(\operatorname{Frob}_{l}\right)=\left(\begin{array}{ll}0 & 1 \\ 1 & 0\end{array}\right)$ if $\left(\frac{d}{l}\right)=-1$, and $\rho_{\chi}\left(\operatorname{Frob}_{l}\right)=\left(\begin{array}{cc}\chi\left(\operatorname{Frob}_{\Lambda}\right) & 0 \\ 0 & \chi^{\sigma}\left(\operatorname{Frob}_{\Lambda}\right)\end{array}\right)$ if $\left(\frac{d}{l}\right)=1$ and $l \mathcal{O}_{K}=\Lambda \sigma(\Lambda)$. This explicit description makes it obvious that the determinant of $\rho_{\chi}$ is the Legendre symbol $l \mapsto\left(\frac{d}{l}\right)$.

Since the kernel of $\chi$ corresponds to a subfield of the Hilbert class field of $K$, simple computations in the class group of $K$ allow one to determine which primes split completely. These give examples satisfying the assumptions of Question 1.9 (the Frobenius at $p$ is the identity) if $\rho_{\chi}$ is odd, i.e. if $p=2$ or $d<0$.

We remark that for characters $\chi$ of odd order $n$ the assumption $\chi^{-1}=\chi^{\sigma}$ is not a big restriction, since any character can be written as $\chi=\chi_{1} \chi_{2}$ with $\chi_{1}^{\sigma}=\chi_{1}^{-1}$ and $\chi_{2}^{\sigma}=\chi_{2}$, hence the latter descends to a character of $\operatorname{Gal}(\overline{\mathbb{Q}} / \mathbb{Q})$ and the representation $\rho_{\chi}$ is isomorphic to $\rho_{\chi_{1}} \otimes \chi_{2}$.

All dihedral representations are known to come from eigenforms in the minimal possible weight with level equal to the (outside of $p$ ) conductor of the representation (see [20], Theorem 1).

In the tables we computed the Hecke algebras of odd dihedral representations as above in the following ranges. For each prime $p$ less than 100 and each prime $l$ less than or equal to the largest level occuring in the table for $p$, we chose $d$ as plus or minus $l$ such that $d$ is $1 \bmod 4$ and we let $H$ run through all non-trivial cyclic quotients of the class group of $\mathbb{Q}(\sqrt{d})$ of order coprime to $p$. For each $H$ we chose (unramified) characters $\chi$ of the absolute Galois group of $\mathbb{Q}(\sqrt{d})$ corresponding to $H$, up to Galois conjugacy and up to replacing $\chi$ by its inverse. Then $\chi$ is not the restriction of a character of $\operatorname{Gal}(\overline{\mathbb{Q}} / \mathbb{Q})$. By genus theory the order of $\chi$ is odd, as the class number is, so we necessarily have $\chi^{-1}=\chi^{\sigma}$. We computed the local factor of $\mathbb{T}_{\mathbb{F}_{p}}\left(S_{p}\left(d,\left(\frac{d}{.}\right) ; \mathbb{F}_{p}\right)\right)$ corresponding to $\rho_{\chi}$ if $\rho_{\chi}$ is odd and $p$ is completely split. For the prime $p=2$ we also allowed square-free integers $d$ which are 1 $\bmod 4$ and whose absolute value is less than 5000 .

\subsection{Icosahedral example}

With the help of a list of polynomials provided by Gunter Malle ([15]) a Galois representation of $\operatorname{Gal}(\overline{\mathbb{Q}} / \mathbb{Q})$ with values in $\mathrm{GL}_{2}\left(\overline{\mathbb{F}}_{2}\right)$ which is of prime conductor, completely split at 2 and thus satisfies the assumptions of Question 1.9 and whose image is isomorphic to the icosahedral group $A_{5}$ could be described explicitly. The modular forms in weight 2 predicted by Serre's conjecture were found and the corresponding Hecke algebra turned out to have Gorenstein defect equal to 2 .

Let $f \in \mathbb{Z}[X]$ be an irreducible polynomial of degree 5 whose Galois group, i.e. the Galois group of the normal closure $L$ of $K=\mathbb{Q}[X] /(f)$, is isomorphic to $A_{5}$. We assume that $K$ is unramified at 2,3 and 5 . We have the Galois representation

$$
\rho_{f}: \operatorname{Gal}(\overline{\mathbb{Q}} / \mathbb{Q}) \rightarrow \operatorname{Gal}(L / \mathbb{Q}) \cong A_{5} \cong \mathrm{SL}_{2}\left(\mathbb{F}_{4}\right)
$$


We now determine its conductor and its traces. Let $p$ be a ramified prime. As the ramification is tame, the image of the inertia group $\rho_{f}\left(I_{p}\right)$ at $p$ is cyclic of order 2,3 or 5 . In the first case, the image of a decomposition group $\rho_{f}\left(D_{p}\right)$ at $p$ is either equal to $\rho_{f}\left(I_{p}\right)$ or equal to $\mathbb{Z} / 2 \mathbb{Z} \times \rho_{f}\left(I_{p}\right)$. If the order of $\rho_{f}\left(I_{p}\right)$ is odd and $\rho_{f}\left(I_{p}\right)=\rho_{f}\left(D_{p}\right)$, then any completion of $L$ at the unique prime above $p$ is totally ramified and cyclic of degree $\# \rho_{f}\left(I_{p}\right)$, hence contained in $\mathbb{Q}_{p}\left(\zeta_{p}\right)$ for $\zeta_{p}$ a primitive $p$-th root of unity. It follows that $p$ is congruent to $1 \bmod \# \rho_{f}\left(I_{p}\right)$. If the order of $\rho_{f}\left(I_{p}\right)$ is odd, but $\rho_{f}\left(I_{p}\right)$ is not equal to $\rho_{f}\left(D_{p}\right)$, then $\rho_{f}\left(D_{p}\right)$ is a dihedral group and the completion of $L$ at a prime above $p$ has a unique unramified quadratic subfield $S$. Thus, we have the exact sequence

$$
0 \rightarrow \rho_{f}\left(I_{p}\right) \rightarrow \rho_{f}\left(D_{p}\right) \rightarrow \operatorname{Gal}\left(S / \mathbb{Q}_{p}\right) \rightarrow 0
$$

On the one hand, it is well-known that the conjugation by a lift of the Frobenius element of $\operatorname{Gal}\left(S / \mathbb{Q}_{p}\right)$ acts on $\rho_{f}\left(I_{p}\right)$ by raising to the $p$-th power. On the other hand, as the action is non-trivial it also corresponds to inversion on $\rho_{f}\left(I_{p}\right)$, since the only elements of order 2 in $(\mathbb{Z} / 3 \mathbb{Z})^{\times}$and $(\mathbb{Z} / 5 \mathbb{Z})^{\times}$ are -1 . As a consequence, $p$ is congruent to $-1 \bmod \# \rho_{f}\left(I_{p}\right)$ in this case.

We hence have the following cases.

(1) Suppose $p \mathcal{O}_{K}=\mathfrak{P}^{5}$. Then $p \equiv \pm 1 \bmod 5$.

(a) If $p \equiv 1 \bmod 5$, then $\left.\rho_{f}\right|_{I_{p}} \sim\left(\begin{array}{cc}\chi & 0 \\ 0 & \chi^{-1}\end{array}\right)$ with $\chi$ a totally ramified character of $\operatorname{Gal}\left(\overline{\mathbb{Q}}_{p} / \mathbb{Q}_{p}\right)$ of order 5 .

(b) If $p \equiv-1 \bmod 5$, then $\rho_{f}\left(D_{p}\right)$ is the dihedral group with 10 elements.

(2) Suppose $p \mathcal{O}_{K}=\mathfrak{P}^{3} \mathfrak{Q} \mathfrak{R}$ or $p \mathcal{O}_{K}=\mathfrak{P}^{3} \mathfrak{Q}$.

(a) If $p \equiv 1 \bmod 3$, then $\left.\rho_{f}\right|_{I_{p}} \sim\left(\begin{array}{cc}\chi & 0 \\ 0 & \chi^{-1}\end{array}\right)$ with $\chi$ a totally ramified character of $\operatorname{Gal}\left(\overline{\mathbb{Q}}_{p} / \mathbb{Q}_{p}\right)$ of order 3 .

(b) If $p \equiv-1 \bmod 3$, then $\rho_{f}\left(D_{p}\right)$ is the dihedral group with 6 elements.

(3) Suppose that $p$ is ramified, but that we are neither in Case (1) nor in Case (2). Then $\left.\rho_{f}\right|_{I_{p}} \sim\left(\begin{array}{ll}1 & 1 \\ 0 & 1\end{array}\right)$.

By the definition of the conductor at $p$ it is clear that it is $p^{2}$ in Cases (1) and (2) and $p$ in Case (3). However, in Cases (1)(a) and (2)(a) one can choose a character $\epsilon$ of Gal $(\overline{\mathbb{Q}} / \mathbb{Q})$ of the same order as $\chi$ whose restriction to $D_{p}$ gives the character $\chi$. If one twists the representation $\rho_{f}$ by $\epsilon$ one finds also in these cases that the conductor at $p$ is $p$.

An inspection of the conjugacy classes of the group $\mathrm{SL}_{2}\left(\mathbb{F}_{4}\right)$ shows that the traces of $\rho_{f}$ twisted by some character $\epsilon$ of $\mathrm{Gal}(\overline{\mathbb{Q}} / \mathbb{Q})$ are as follows. Let $l$ be an unramified prime.

- If the order of $\operatorname{Frob}_{l}$ is 5 , then the trace at $\operatorname{Frob}_{l}$ is $\epsilon\left(\operatorname{Frob}_{l}\right) w$ where $w$ is a root of the polynomial $X^{2}+X+1$ in $\mathbb{F}_{2}[X]$.

- If the order of Frob ${ }_{l}$ is 3 , then the trace at Frob $l$ is $\epsilon\left(\right.$ Frob $\left._{l}\right)$.

- If the order of Frob $l$ is 1 or 2, then the trace at $\operatorname{Frob}_{l}$ is 0. 
These statements allow the easy identification of the modular form belonging to an icosahedral representation.

We end this section with some remarks on our icosahedral example. It was obtained using the polynomial $x^{5}-x^{4}-79 x^{3}+225 x^{2}+998 x-3272$. The corresponding table entry is:

\begin{tabular}{||c|c|c|c|c|c|c|c|c|c||}
\hline Level & Wt & ResD & Dim & EmbDim & NilO & GorDef & \#Ops & $\#(\mathrm{p}<\mathrm{HB})$ & Gp \\
\hline 89491 & 2 & 2 & 12 & 4 & 3 & 2 & 4 & 1746 & $A_{5}$ \\
\hline
\end{tabular}

Hence, in level 89491 and weight 2 there is a single eigenform $g$ mod 2 up to Galois conjugacy whose first couple of $q$-coefficients agree with the traces of a twist of the given icosahedral Galois representation. From this one can deduce that the Galois representation $\rho_{g}$ of $g$ has an icosahedral image and is only ramified at 89491. As weight and level lowering are not known in our case, we cannot prove that $\rho_{g}$ coincides with a twist of the given one. It might, however, be possible to exclude the existence of two distinct icosahedral extensions of the rationals inside $\mathbb{C}$ that ramify only at 89491 by consulting tables. According to Malle, the icosahedral extension used has smallest discriminant among all totally real $A_{5}$-extensions of the rationals in which 2 splits completely.

\section{Further results and questions}

In this section we present some more computational observations for Hecke algebras under the assumptions of Question 1.9, which lead us to ask some more questions.

\section{On the dimension of the Hecke algebra}

From the data, we see that many even integers appear as dimensions of the $\mathbb{T}_{\mathfrak{m}}$. We know that the dimension must be at least 4 , as this is the dimension of the smallest non-Gorenstein algebra which can appear in our case. This extends the results of [14], where the dimensions of the Hecke algebras $\mathbb{T}_{\mathbb{Z} \rightarrow \mathbb{F}_{2}}\left(S_{2}\left(\Gamma_{0}(431)\right)\right)$ and $\mathbb{T}_{\mathbb{Z} \rightarrow \mathbb{F}_{2}}\left(S_{2}\left(\Gamma_{0}(503)\right)\right)$ localised at the non-Gorenstein maximal ideals are shown to be 4 .

In this table we see exactly how many times each dimension appears in our data. We observe that every even integer between 4 and 32 appears, and that the largest dimension is 60 . The most common dimension is 4 , which appears about half of the time. However, as the dimension of the Hecke algebra attached to $S_{k}\left(\Gamma_{1}(N)\right)$ increases with $N$ and with $k$, this may be an artifact of the data being collected for "small" levels $N$ and primes $p$.

\begin{tabular}{|l|l|l|l|l|l|l|l|l|l|l|}
\hline Dimension & 4 & 6 & 8 & 10 & 12 & 14 & 16 & 18 & 20 & 22 \\
\hline Number of algebras & 206 & 58 & 25 & 3 & 24 & 6 & 20 & 3 & 12 & 3 \\
\hline \hline Dimension & 24 & 26 & 28 & 30 & 32 & 36 & 40 & 46 & 56 & 60 \\
\hline Number of algebras & 5 & 4 & 2 & 1 & 2 & 2 & 4 & 1 & 2 & 1 \\
\hline
\end{tabular}

It seems reasonable that there should be infinitely many cases with dimension 4 , and plausible that every even integer greater than or equal to 4 should appear as a dimension infinitely many times. 
From the tables, we see that dimension 4 algebras appear at very high levels, so they do not appear to be becoming rare as the dimension increases, but this may, of course, be an artifact of our data.

We note that not every example that arises from an elliptic curve in characteristic $p=2$ has Hecke algebra with dimension 4; for example the algebra $\mathbb{T}_{\mathbb{Z} \rightarrow \mathbb{F}_{2}}\left(S_{2}\left(\Gamma_{0}(2089)\right)\right)$ localised at its nonGorenstein maximal ideal has dimension 18. In level 18097 there is a dimension 36 example arising from an elliptic curve.

\section{On the residue degree}

We will now solve an easy aspect of the question of the possible structures of non-Gorenstein local algebras occurring as local Hecke algebras. We assume for the couple of lines to follow the Generalised Riemann Hypothesis (GRH).

We claim that then the residue degrees of $\mathbb{T}_{\mathfrak{m}}$ (in the notation of Question 1.9) are unbounded, if we let $p$ and $N$ run through the primes such that $p \neq N$ and $N$ is congruent to 3 modulo 4 .

For, class groups of imaginary quadratic fields $\mathbb{Q}(\sqrt{-N})$ have arbitrarily large cyclic factors of odd order, as the exponent of these class groups is known to go to infinity as $N$ does, by the main result of [4], which assumes GRH. So the discussion on dihedral forms in Section 4 immediately implies the claim.

\section{On the embedding dimension}

One can ask whether the embedding dimension of the local Hecke algebras in the situation of Question 1.9 is bounded, if we allow $p$ and $N$ to vary. This, however, seems to be a difficult problem. The embedding dimensions occuring in our tables are 3 (299 times), 4 (78 times) and 5 (7 times).

The embedding dimension $d$ is related to the number of Hecke operators needed to generate the local Hecke algebra, in the sense that at least $d$ Hecke operators are needed. Probably, $d$ Hecke operators can be found that do generate, but they need not be the first $d$ prime Hecke operators, of course. However, as our tables suggest, in most cases the actual computations were done using very few operators, and there are 99 of the 384 cases when the computation already finished after $d$ operators.

\section{Acknowledgements}

The authors would like to thank Kevin Buzzard for informing them about the level 23 and weight 59 example, for many helpful conversations and communications, and for his comments on a first draft of this paper. They would like to thank Amod Agashe for comments on an early version and William Stein for the use of his computers MECCAH and NERON for computations; without these, they would not have been able to pursue these computations as far as they did. The authors are grateful to Gunter Malle for providing them with a very useful list of $A_{5}$-polynomials. The first author would also like 
to thank Edray Goins for helpful conversations. The second author is endebted to Bas Edixhoven for very enlightening explanations.

\section{References}

[1] Amod Agashe, Kenneth A. Ribet, and William A. Stein. The Modular Degree, Congruence Primes and Multiplicity One. http://modular.math.washington.edu/papers/ars-congruence/, 2006.

[2] W. Bosma, J. Cannon, and C. Playoust. The Magma algebra system I: The user language. J. Symb. Comp., 24(3-4):235-265, 1997. http://magma.maths.usyd.edu.au.

[3] Nigel Boston, Hendrik W. Lenstra, Jr., and Kenneth A. Ribet. Quotients of group rings arising from two-dimensional representations. C. R. Acad. Sci. Paris Sér. I Math., 312(4):323-328, 1991.

[4] David W. Boyd and H. Kisilevsky. On the exponent of the ideal class groups of complex quadratic fields. Proc. Amer. Math. Soc., 31:433-436, 1972.

[5] K. Buzzard. Appendix to Lectures on Serre's Conjectures, [18]. In IAS/ Park City Mathematics Institute Lecture Series, 1999.

[6] Fred Diamond and John Im. Modular forms and modular curves. In Seminar on Fermat's Last Theorem (Toronto, ON, 1993-1994), volume 17 of CMS Conf. Proc., pages 39-133. Amer. Math. Soc., Providence, RI, 1995.

[7] Bas Edixhoven. The weight in Serre's conjectures on modular forms. Invent. Math., 109(3):563594, 1992.

[8] Bas Edixhoven. Serre's conjecture. In Modular forms and Fermat's last theorem (Boston, MA, 1995), pages 209-242. Springer, New York, 1997.

[9] Bas Edixhoven. Comparison of integral structures on spaces of modular forms of weight two, and computation of spaces of forms mod 2 of weight one. J. Inst. Math. Jussieu, 5(1):1-34, 2006. With appendix A (in French) by Jean-François Mestre and appendix B by Gabor Wiese.

[10] David Eisenbud. Commutative algebra, volume 150 of Graduate Texts in Mathematics. Springer-Verlag, New York, 1995. With a view toward algebraic geometry.

[11] Matthew Emerton. Supersingular elliptic curves, theta series and weight two modular forms. $J$. Amer. Math. Soc., 15(3):671-714 (electronic), 2002.

[12] Matthew Emerton, Robert Pollack, and Tom Weston. Variation of Iwasawa invariants in Hida families. Invent. Math., 163(3):523-580, 2006. 
[13] B. Gross. A tameness criterion for Galois representations associated to modular forms $(\bmod p)$. Duke Math. Journal, 61(2):445-517, 1990.

[14] L. J. P. Kilford. Some non-Gorenstein Hecke algebras attached to spaces of modular forms. $J$. Number Theory, 97(1):157-164, 2002.

[15] G. Malle. Personal communication. 2006.

[16] B. Mazur. Modular curves and the Eisenstein ideal. Inst. Hautes Études Sci. Publ. Math., (47):33-186 (1978), 1977.

[17] Kenneth A. Ribet. Multiplicities of Galois representations in Jacobians of Shimura curves. In Festschrift in honor of I. I. Piatetski-Shapiro on the occasion of his sixtieth birthday, Part II (Ramat Aviv, 1989), volume 3 of Israel Math. Conf. Proc., pages 221-236. Weizmann, Jerusalem, 1990.

[18] Kenneth A. Ribet and William A. Stein. Lectures on Serre's conjectures. In Arithmetic algebraic geometry (Park City, UT, 1999), volume 9 of IAS/Park City Math. Ser., pages 143-232. Amer. Math. Soc., Providence, RI, 2001.

[19] William A. Stein. Explicitly Computing Modular Forms. Textbook to appear, 2006.

[20] Gabor Wiese. Dihedral Galois representations and Katz modular forms. Doc. Math., 9:123-133 (electronic), 2004.

[21] Gabor Wiese. On modular symbols and the cohomology of Hecke triangle surfaces. arXiv:math.NT/0511113, 2005.

[22] Gabor Wiese. On the faithfulness of parabolic cohomology as a Hecke module over a finite field. Accepted for publication in J. für die reine und angewandte Mathematik. arXiv:math.NT/0511115, 2005.

[23] Gabor Wiese. Multiplicities of Galois representations of weight one. Preprint, 2006.

[24] Andrew Wiles. Modular elliptic curves and Fermat's last theorem. Ann. of Math. (2), 141(3):443-551, 1995.

L. J. P. Kilford

Mathematics Institute

Oxford University

24-29 St Giles'

Oxford OX1 3LB

United Kingdom

l.kilfordegmail.com
Gabor Wiese

NWF 1-Mathematik

Universität Regensburg

D-93040 Regensburg

Germany

gabor@pratum. net

http://maths.pratum.net 


\title{
A The Magma package HeckeAlgebra (by Gabor Wiese)
}

\author{
Abstract \\ This is a short manual for the MAGMA package HeckeAlgebra, which can be downloaded \\ from the author's webpage. The author would like to thank Lloyd Kilford for very helpful sug- \\ gestions.
}

\section{A.1 Example}

The following example explains the main functions of the package. Let us suppose that the file HeckeAlgebra.mg is stored in the current path. We first attach the package.

> Attach("HeckeAlgebra.mg");

We want the package to be silent, so we put:

> SetVerbose ("HeckeAlgebra",false);

If we would like more information on the computations being performed, we should have put the value true. Since we want to store the data to be computed in a file, we now create the file.

$>$ my_file := "datafile";

$>$ CreateStorageFile(my_file);

Next, we would like to compute the Hecke algebras of the dihedral eigenforms of level 2039 over extensions of $\mathbb{F}_{2}$. First, we create a list of such forms.

$>$ dih $:=$ DihedralForms $(2039$ : ListOfPrimes $:=[2]$, completely_split $:=$ false);

Now, we compute the corresponding Hecke algebras, print part of the computed data in a human readable format, and finally save the data to our file.

$>$ for $f$ in dih do

for> ha := HeckeAIgebras(f);

for> HeckeAlgebraPrint1(ha);

for>StoreData(my_file, ha);

for> end for;

Level 2039

Weight 2

Characteristic 2

Gorenstein defect 0

Dimension 1

Number of operators used 3

Primes lt Hecke bound 68

Residue degree 2

Level 2039

Weight 2

Characteristic 2

Gorenstein defect 2

Dimension 6

Number of operators used 4

Primes lt Hecke bound 68 
Residue degree 2

Level 2039

Weight 2

Characteristic 2

Gorenstein defect 0

Dimension 1

Number of operators used 3

Primes lt Hecke bound 68

Residue degree 6

Level 2039

Weight 2

Characteristic 2

Gorenstein defect 0

Dimension 1

Number of operators used 3

Primes lt Hecke bound 68

Residue degree 4

Level 2039

Weight 2

Characteristic 2

Gorenstein defect 0

Dimension 1

Number of operators used 3

Primes lt Hecke bound 68

Residue degree 4

Level 2039

Weight 2

Characteristic 2

Gorenstein defect 0

Dimension 1

Number of operators used 3

Primes lt Hecke bound 68

Residue degree 12

Level 2039

Weight 2

Characteristic 2

Gorenstein defect 0

Dimension 1

Number of operators used 3 
With the function DihedralForms one may also compute exclusively representations that are completely split in the characteristic. The default is completely_split $:=$ true. By the option bound we indicate primes up to which bound should be used as the characteristic. The following example illustrates this.

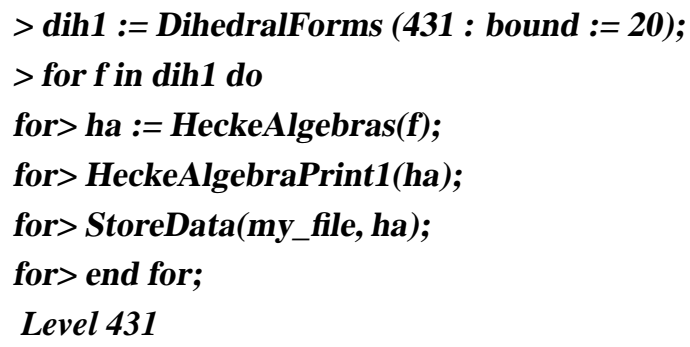

Level 431

Weight 11

Characteristic 11

Gorenstein defect 2

Dimension 4

Number of operators used 5

Primes lt Hecke bound 77

Residue degree 3

One can also compute icosahedral modular forms over extensions of $\mathbb{F}_{2}$, starting from an integer polynomial with Galois group $A_{5}$, as follows.

$>\boldsymbol{R}<\boldsymbol{x}>$ := PolynomialRing(Integers ()$)$;

$>$ pol : $=x^{\wedge} 5-x^{\wedge} 4-780 * x^{\wedge} 3-1795 * x^{\wedge} 2+3106 * x+344$

$>\boldsymbol{f}:=\operatorname{A5Form}($ pol $)$;

With this kind of icosahedral examples one has to pay attention to the conductor, as it can be huge. This polynomial has prime conductor. But conductors need not be square-free, in general.

$>$ print Modulus(f'Character);

1951

So it's reasonable. We do the computation.

> ha := HeckeAlgebras(f);

> HeckeAlgebraPrint1(ha);

Level 1951 
Weight 2

Characteristic 2

Gorenstein defect 0

Dimension 3

Number of operators used 3

Primes lt Hecke bound 66

Residue degree 4

Level 1951

Weight 2

Characteristic 2

Gorenstein defect 0

Dimension 6

Number of operators used 3

Primes lt Hecke bound 66

Residue degree 4

There are two forms, which is okay, since they come from a weight one form in two different ways and this case is not exceptional. We now save them, as always.

> StoreData(my_file, ha);

It is also possible to compute all forms at a given character and weight.

$>$ eps := DirichletGroup $(229, G F(2)) .1$;

$>$ ha := HeckeAlgebras(eps,2);

> HeckeAlgebraPrint1(ha);

Level 229

Weight 2

Characteristic 2

Gorenstein defect 0

Dimension 1

Number of operators used 12

Primes lt Hecke bound 12

Residue degree 1

Level 229

Weight 2

Characteristic 2

Gorenstein defect 0

Dimension 2

Number of operators used 12

Primes lt Hecke bound 12

Residue degree 2

Level 229

Weight 2 


\section{Characteristic 2}

Gorenstein defect 0

Dimension 4

Number of operators used 12

Primes lt Hecke bound 12

Residue degree 1

Level 229

Weight 2

Characteristic 2

Gorenstein defect 0

Dimension 2

Number of operators used 12

Primes lt Hecke bound 12

Residue degree 5

\section{> StoreData(my_file,ha);}

Next, we illustrate how one reloads what has been saved. One would like to type: Ioad my_file; but that does not work. One has to do it as follows.

$>$ load "datafile";

$>$ mf := RecoverData(LoadIn,LoadInReI);

Now, $\boldsymbol{m} \boldsymbol{f}$ contains a list of all algebra data computed before. There's a rather concise printing function, displaying part of the information, namely HeckeAlgebraPrint(mf);

One can also create a LaTeX longtable. The entries can be chosen in quite a flexible way. The standard usage is the following.

> HeckeAlgebraLaTeX(mf, "table.tex");

A short LaTeX file displaying the table is the following:

\documentclass [11pt] \{article\}

\usepackage \{longtable\}

$\backslash$ begin \{document $\}$

$\backslash$ input $\{$ table

\end \{document }

The table we created is this one:

\begin{tabular}{||c|c|c|c|c|c|c|c|c|c||}
\hline Level & Wt & ResD & Dim & EmbDim & NilO & GorDef & \#Ops & $\#(\mathrm{p}<\mathrm{HB})$ & $\mathrm{Gp}$ \\
\hline 2039 & 2 & 2 & 1 & 0 & 0 & 0 & 3 & 68 & $D_{3}$ \\
2039 & 2 & 2 & 6 & 3 & 2 & 2 & 4 & 68 & $D_{5}$ \\
2039 & 2 & 6 & 1 & 0 & 0 & 0 & 3 & 68 & $D_{9}$ \\
2039 & 2 & 4 & 1 & 0 & 0 & 0 & 3 & 68 & $D_{15}$ \\
2039 & 2 & 4 & 1 & 0 & 0 & 0 & 3 & 68 & $D_{15}$ \\
2039 & 2 & 12 & 1 & 0 & 0 & 0 & 3 & 68 & $D_{45}$ \\
2039 & 2 & 12 & 1 & 0 & 0 & 0 & 3 & 68 & $D_{45}$ \\
431 & 2 & 1 & 4 & 3 & 1 & 2 & 6 & 20 & $D_{3}$ \\
431 & 11 & 3 & 4 & 3 & 1 & 2 & 5 & 77 & $D_{7}$ \\
1951 & 2 & 4 & 3 & 1 & 2 & 0 & 3 & 66 & $A_{5}$ \\
\hline
\end{tabular}




\begin{tabular}{||c|c|c|c|c|c|c|c|c|c||}
\hline Level & Wt & ResD & Dim & EmbDim & NilO & GorDef & \#Ops & $\#(\mathrm{p}<\mathrm{HB})$ & Gp \\
\hline 1951 & 2 & 4 & 6 & 2 & 3 & 0 & 3 & 66 & $A_{5}$ \\
229 & 2 & 1 & 1 & 0 & 0 & 0 & 12 & 12 & \\
229 & 2 & 2 & 2 & 1 & 1 & 0 & 12 & 12 & \\
229 & 2 & 1 & 4 & 1 & 3 & 0 & 12 & 12 & \\
229 & 2 & 5 & 2 & 1 & 1 & 0 & 12 & 12 & \\
\hline \hline
\end{tabular}

In the examples of level 229 the image of the Galois representation as an abstract group is not know. That is due to the fact that we created these examples without specifying the Galois representation in advance.

It is possible to compute arbitrary Hecke operators on the local Hecke factors generated by HeckeAlgebras $(\cdot)$, as the following example illustrates.

$>$ A,B,M,C := HeckeAlgebras(DirichletGroup(253,GF(2)).1,2 : over_residue_field := true); Suppose that we want to know the Hecke operator $T_{17}$ on the 4 th local factor.

$>\mathbf{i}:=4$

$>T:=$ BaseChange(HeckeOperator(M,17),C[i]);

The coefficients are the eigenvalues (only one):

$>$ Eigenvalues $(T)$;

$\{<\$ .1 \wedge 5,8>\}$

Let us remember the eigenvalue.

$>$ e := SetToSequence(Eigenvalues $(T))[1][1]$;

In order to illustrate the option over_residue_field, we also compute the following:

$>$ A1,B1,M1,C1 := HeckeAlgebras(DirichletGroup(253,GF(2)).1,2 : over_residue_field := false);

> T1 := BaseChange(HeckeOperator(M1,17),C1[i]);

$>$ Eigenvalues(T1);

\}

The base field is strictly smaller than the residue field in this example and the operator $\mathbf{T 1}$ cannot be diagonalised over the base field. We check that $\mathbf{e}$ is nevertheless a zero of the minimal polynomial of $\mathbf{T 1}$.

> Evaluate(MinimalPolynomial(T1),e);

0

The precise usage of the package is described in the following sections.

\section{A.2 Hecke algebra computation}

\section{A.2.1 The modular form format}

In the package, modular forms are often represented by the following record.

ModularFormFormat $:=$ recformat $<$

$\begin{array}{ll}\text { Character } & \text { : GrpDrchEIt, } \\ \text { Weight } & \text { : RngIntElt, } \\ \text { CoefficientFunction } & \text { : Map, }\end{array}$




$\begin{array}{ll}\text { ImageName } & : \text { MonStgElt, } \\ \text { Polynomial } & : \text { RngUPolElt }\end{array}$

>;

The fields Character and Weight have the obvious meaning. Sometimes, the image of the associated Galois representation is known as an abstract group. Then that name is recorded in ImageName, e.g. $\boldsymbol{A} \_5$ or $\boldsymbol{D} \_3$. In some cases, a polynomial is known whose splitting field is the number field cut out by the Galois representation. Then the polynomial is stored in Polynomial. The cases in which polynomials are known are usually icosahedral ones. The CoefficientFunction is a function from the integers to a polynomial ring. For all primes $l$ different from the characteristic and not dividing the level of the modular form (i.e. the modulus of the Character), the coefficient function should return the minimal polynomial of the $l$-th coefficient in the $q$-expansion of the modular form in question.

\section{A.2.2 Dihedral modular forms}

Eigenforms whose associated Galois representations take dihedral groups as images provide an important source of examples, in many contexts. These eigenforms are called dihedral. The big advantage is that their Galois representation, and hence their $q$-coefficients, can be computed using class field theory. That enables one to exhibit Galois representations in the context of modular forms with certain number theoretic properties. The property for which these functions were initially created is that the representations should be unramified in the characteristic, say $p$, and that $p$ is completely split in the number field cut out by the representation.

We consider dihedral representations whose determinant is the Legendre symbol of a quadratic field $\mathbb{Q}(\sqrt{N})$. The representations produced by the functions to be described are obtained by induction of an unramified character $\chi$ of $\mathbb{Q}(\sqrt{N})$ whose conjugate by the non-trivial element of the Galois group of $\mathbb{Q}(\sqrt{N})$ over $\mathbb{Q}$ is assumed to be $\chi^{-1}$.

intrinsic GetLegendre ( $\mathrm{N}$ :: RngIntElt, $\mathrm{K}$ :: FldFin ) -> GrpDrchEIt

For an odd positive integer $N$, this function returns the element of $\operatorname{DirichletGroup}(\boldsymbol{A b s}(\mathbf{N}), \boldsymbol{K})$ (with $\boldsymbol{K}$ a finite field of characteristic different from 2) which corresponds to the Legendre symbol $p \mapsto\left(\frac{ \pm N}{p}\right)$. If $\mathbf{N}$ is $1 \bmod 4$ the sign is +1 , and -1 otherwise.

intrinsic DihedralForms (N :: RngIntElt :

ListOfPrimes $:=[]$, bound $:=100$, odd_only $:=$ true, quad_disc $:=0$, completely_split $:=$ true, all_conjugacy_classes $:=$ true ) $->$ Rec

This function computes all modular forms (in the sense of Section A.2.1) of level $\mathbf{N}$ and weight $p$ over a finite field of characteristic $p$ that come from dihedral representations whose determinant is the Legendre symbol of the quadratic field $K=\mathbb{Q}(\sqrt{ \pm \text { quad_disc }})$ and which are obtained by induction of an unramified character of $K$. If quad_disc is $1 \bmod 4$ the sign is +1 , and -1 otherwise. If quad_disc is 0 , the value of $\mathbf{N}$ is used. If the option completely_split is set, only those representations are returned which are completely split at $p$. If the option ListOfPrimes is assigned a non-empty list of primes, only those primes are considered as the characteristic. If it is the empty set, all primes $p$ up to the bound are taken into consideration. If the option odd_only is true, only odd Galois representations are returned. If the option all_conjugacy_classes is true, each unramified character as above up to Galois conjugacy and up to taking inverses is used. 
Otherwise, a single choice is made. That there may be non-conjugate characters cutting out the same number field is due to the fact that there may be non-conjugate elements of the same order in the multiplicative group of a finite field.

\section{A.2.3 Icosahedral modular forms}

Eigenforms whose attached Galois representations take the group $A_{5}$ as projective images are called icosahedral. Since extensive tables of $A_{5}$-extensions of the rationals are available, one can consider icosahedral Galois representations which one knows very well. That allows one to test certain conjectures concerning modular forms on icosahedral ones.

We note the isomorphism $A_{5} \cong \mathrm{SL}_{2}\left(\mathbb{F}_{4}\right)$. Thus, $A_{5}$-extentions of the rationals give rise to icosahedral Galois representations in characteristic 2 which (should) come from modular forms mod 2. It would also be possible to use certain other primes, but this has not been implemented.

\section{intrinsic A5Form (f :: RngUPolElt) -> Rec}

Returns the icosahedral form in characteristic 2 and weight 2 of smallest predicted level corresponding to the polynomial $\boldsymbol{f}$ which is expected to be of degree 5 and whose Galois group is supposed to be $A_{5}$. No checks about $\boldsymbol{f}$ are performed.

\section{A.2.4 The Hecke algebra format}

The data concerning the Hecke algebra of an eigenform that is computed by the function HeckeAlgebras is a record of the following form.

AlgebraData := recformat $<$

$\begin{array}{ll}\text { Level } & : \text { RngIntElt, } \\ \text { Weight } & : \text { RngIntElt, } \\ \text { Characteristic } & : \text { RngIntElt, } \\ \text { BaseFieldDegree } & : \text { RngIntElt, } \\ \text { CharacterOrder } & : \text { RngIntElt, } \\ \text { CharacterConductor } & : \text { RngIntEIt, } \\ \text { CharacterIndex } & : \text { RngIntElt, } \\ \text { AlgebraFieldDegree } & : \text { RngIntEIt, } \\ \text { ResidueDegree } & : \text { RngIntEIt, } \\ \text { Dimension } & : \text { RngIntEIt, } \\ \text { GorensteinDefect } & : \text { RngIntEIt, } \\ \text { EmbeddingDimension } & : \text { RngIntEIt, } \\ \text { NilpotencyOrder } & : \text { RngIntElt, } \\ \text { Relations } & : \text { Tup, } \\ \text { NumberGenUsed } & : \text { RngIntEIt, } \\ \text { ImageName } & : \text { MonStgEIt, } \\ \text { Polynomial } & : \text { RngUPolEIt }\end{array}$

$>$;

Level and Weight have the obvious meaning. Let $K$ be the base field for the space of modular symbols used. It is (expected to be) a finite field. Then Characteristic is the character- 
istic of $K$ and BaseFieldDegree is the degree of $K$ over its prime field. The entries CharacterOrder, CharacterConductor and CharacterIndex concern the Dirichlet character for which the modular symbols have been computed. The latter field is the index of the character in Elements(DirichletGroup( $\cdot$ )). Note that that might change between different versions of Magma. The fields ResidueDegree (over the prime field), Dimension and GorensteinDefect have their obvious meaning for the Hecke algebra in question. The tuple

\section{<AlgebraFieldDegree, EmbeddingDimension, NilpotencyOrder, Relations>}

are data from which AffineAlgebra can recreate the Hecke algebra up to isomorphism. NumberGenUsed indicates the number of generators used by the package for the computation of the Hecke algebra. This number is usually much smaller than the Sturm bound. ImageName and Polynomial have the same meaning as in the record ModularFormFormat.

\section{A.2.5 Hecke algebras}

intrinsic HeckeAlgebras (eps :: GrpDrchElt, weight :: RngIntElt :

UserBound $:=0$, first_test $:=3$, test_interval $:=1$, when_test_p $:=3$,

when_test_bad $:=4$, test_sequence $:=[]$, dimension_factor $:=2$,

ms_space $:=0$, cuspidal $:=$ true, DegreeBound $:=0$, OperatorList $:=[]$,

over_residue_field $:=$ true, $t$ try_minimal $:=$ true, force_local $:=$ false,

) -> SeqEnum, SeqEnum, ModSym, Tup, Tup

intrinsic HeckeAlgebras ( $t$ :: Rec :

UserBound $:=0$, first_test $:=3$, test_interval $:=1$, when_test_p $:=3$,

when_test_bad $:=4$, test_sequence $:=[]$, dimension_factor $:=2$,

ms_space $:=0$, cuspidal $:=$ true, DegreeBound $:=0$, OperatorList $:=[]$,

over_residue_field $:=$ true, try_minimal $:=$ true, force_local $:=$ false,

) -> SeqEnum, SeqEnum, ModSym, Tup, Tup

These functions compute all local Hecke algebras (up to Galois conjugacy) in the specified weight for the given Dirichlet character eps, respectively those corresponding to the modular form $\boldsymbol{t}$ given by a record of type ModularFormFormat. The functions return 5 values $\boldsymbol{A}, \boldsymbol{B}, \boldsymbol{C}, \boldsymbol{D}, \boldsymbol{E}$. A contains a list of records of type AlgebraData describing the local Hecke algebra factors. $\boldsymbol{B}$ is a list containing the local Hecke algebra factors as matrix algebras. $\boldsymbol{C}$ is the space of modular symbols used in the computations. $\boldsymbol{D}$ is a tuple containing the base change tuples describing the local Hecke factors. We need to know $\boldsymbol{D}$ in order to compute matrices representing Hecke operators for the local factor. Finally, $\boldsymbol{E}$ contains a tuple consisting of all Hecke operators computed so far for each local factor of the Hecke algebra.

The usage in practice is described in the example at the beginning of this manual. We now explain the different options in detail.

The modular symbols space to be used in the computations can be determined as follows. The option ms_space can be set to the values 1 (the plus-space), -1 (the minus-space) and 0 (the full space). Whether the restriction to the cuspidal subspace is taken, is determined by cuspidal. It is not necessary to pass to the cuspidal subspace, for example, if a cusp form is given by a coefficient function (see the description of the record ModularFormFormat). 
In some cases, a list of Hecke operators on the modular symbols space in question may already have been computed. In order to prevent MAGMA from redoing their computations, they may be passed on to the function using the option OperatorList.

Often, one wants to compute the local Hecke algebra of a modular form whose degree of the coefficient field over its prime field is known, e.g. in the case of an icosahedral form in characteristic 2 for the trivial Dirichlet character the coefficient field is $\mathbb{F}_{4}$. By assigning a positive value to the option DegreeBound the function will automatically discard any systems of eigenvalues beyond that bound, which speeds up the computations. One must be a bit careful with this option, as there may be cases when the bound may not be respected at "bad primes". But it usually suffices to take twice the degree of the coefficient field, e.g. one chooses DegreeBound := 4 in the icosahedral example just described. If no system of eigenvalues should be discarded for degree reasons, one must set DegreeBound :=0 .

All of the options first_test, test_interval, when_test_p,when_test_bad, test_sequence, force_local, dimension_factor and UserBound concern the stop criterion. Theoretically, the Sturm bound (see HeckeBound) tells us up to which bound Hecke operators must be computed in order to be sure that they generate the whole Hecke algebra. In practice, however, the algorithm can often determine itself when enough Hecke operators have been computed to generate the algebra. That number is usually much smaller than the Sturm bound. The Sturm bound can be overwritten by assigning a positive number to UserBound

The stop criterion is the following. Let $M$ be the modular symbols space used and $S$ the set of Hecke operators computed so far. Then $M=\bigoplus_{i=1}^{r} M_{i}$ (for some $r$ ) such that each $M_{i}$ is respected by the Hecke operators and the minimal polynomial of each $T \in S$ restricted to $M_{i}$ is a power of an irreducible polynomial (i.e. each $M_{i}$ is a primary space for the action of the algebra generated by all elements of $S$ ). Let $A_{i}$ be the algebra generated by $\left.T\right|_{M_{i}}$ for all $T \in S$. One knows (in many cases, and in all cases of interest) that $A_{i}$ is equal to a direct product of local Hecke algebras if one has the equality

$$
f \times \operatorname{dim}\left(A_{i}\right)=\text { dimension of } M_{i} .
$$

Here, $f$ is given by dimension_factor and should be 1 if the plus-space or the minus space of modular symbols are used, and 2 otherwise. The correct assignment of dimension_factor must be made by hand, whence experimentations are possible. If the stop criterion is not reached, the algorithm terminates at the Hecke bound.

It may happen that, when the stop criterion is reached, one $A_{i}$ is isomorphic to a direct product of more than one local Hecke algebras. If in that case the option force_local is true, the computation of Hecke operators is continued until each $A_{i}$ is isomorphic to a single Hecke factor. If force_local is false, then a fast localisation algorithm is applied to each $A_{i}$. The option is useful, when one expects only a single local Hecke algebra factor, for example, when a modular form is given.

In many cases of interest the Hecke operator $T_{p}$ with $p$ the characteristic is needed in order to generate the whole Hecke algebra. The option when_test_p tells the algorithm at which step to compute $T_{p}$. It is very advisable to choose a small number. In practice, the stop criterion is reached after very few steps, e.g. 5 steps, when $T_{p}$ is computed early. Otherwise, the algorithm often has to continue until $T_{p}$ is computed, although most of the operators before did not change the generated algebra. The option when_test_bad has a similar meaning for the $T_{l}$ for primes $l$ 
dividing the level. However, paying attention to them is only required when the modular form is old at $l$. Moreover, one can assign a list of primes to test_sequence. The algorithm will then start with the Hecke operators indicated by that sequence, and then continue with the others.

The option first_test tells the algorithm at which step the first test for the stop criterion is to be performed. The next test is then carried out after test_interval many steps, and so on. These numbers should be chosen small, too, unless the dimension test takes much time, which is rare, so that one wants to perform it less often, meaning that possibly more Hecke operators than necessary are computed (time consuming).

The option over_residue_field tells the algorithm whether at the end of the computation the local Hecke factors should be base changed to their residue field. If that is done, only one of the conjugate local factors of the base changed algebra is retained.

Finally, the option try_minimal is passed on to AffineAlgebra, when the output is generated. Calling that function with the option set true can sometimes be very time consuming, but makes the output much shorter.

\section{A.2.6 Storage functions}

The package provides functions to store a list whose elements are records of type AlgebraData in a file, and to re-read it. The usage of these functions is explained in the example at the beginning of this manual.

intrinsic CreateStorageFile ( filename :: MonStgElt )

This function prepares the file filename for storing the data.

intrinsic StoreData (filename :: MonStgEIt, forms :: SeqEnum)

This functions appends the list forms of Hecke algebra data to the file filename. That file must have been created by CreateStorageFile.

intrinsic StoreData (filename :: MonStgElt, form :: Rec)

This function appends the Hecke algebra data form to the file filename. That file must have been created by CreateStorageFile.

intrinsic RecoverData (LoadIn :: SeqEnum, LoadInRel :: Tup ) -> SeqEnum

In order to read Hecke algebra data from file "name", proceed as follows:

> load "name";

$>$ readData := RecoverData(LoadIn,LoadInReI).

Then readData will contain a list whose elements are records of type AlgebraData.

\section{A.2.7 Output functions}

intrinsic HeckeAlgebraPrint (ha :: SeqEnum)

intrinsic HeckeAlgebraPrint1 (ha :: SeqEnum)

These functions print part of the data stored in the list ha of records of type AlgebraData in a human readable format.

intrinsic GetLevel (a :: Rec) -> Any

intrinsic GetWeight (a :: Rec) -> Any

intrinsic GetCharacteristic (a :: Rec) -> Any 
intrinsic GetResidueDegree (a :: Rec) -> Any

intrinsic GetDimension (a :: Rec) -> Any

intrinsic GetGorensteinDefect (a :: Rec) -> Any

intrinsic GetEmbeddingDimension (a :: Rec) -> Any

intrinsic GetNilpotencyOrder (a :: Rec) -> Any

intrinsic GetHeckeBound (a :: Rec) -> Any

intrinsic GetPrimesUpToHeckeBound (a :: Rec) -> Any

intrinsic GetNumberOperatorsUsed (a :: Rec) -> Any

intrinsic GetPolynomial (a :: Rec) -> Any

intrinsic GetImageName (a :: Rec) -> Any

These functions return the property of the record a of type AlgebraData specified by the name of the function. If the corresponding attribute is not assigned, the empty string is returned.

intrinsic HeckeAlgebraLaTeX (ha :: SeqEnum, filename :: MonStgElt : which := [

<GetLevel, "Level">, <GetWeight,"Wt">, <GetResidueDegree,"ResD">,

<GetDimension, "Dim">, <GetEmbeddingDimension,"EmbDim">,

<GetNilpotencyOrder,"NilO">, <GetGorensteinDefect,"GorDef">,

$<$ GetNumberOperatorsUsed,"\#Ops">,

<GetPrimesUpToHeckeBound,"\#(p<HB)">, <GetImageName,"Gp"> ] )

This function creates the LaTeX file filename containing a longtable consisting of certain properties of the objects in ha which are supposed to be records of type AlgebraData. The properties to be written are indicated by the list given in the option which consisting of tuples $\langle\mathbf{f}$, name>. Here $\boldsymbol{f}$ is a function that evaluates a record of type AlgebraData to some Magma object which is afterwards transformed into a string using Sprint. Examples for $\boldsymbol{f}$ are the functions GetLevel etc., which are described above. The name will appear in the table header. For a sample usage, see the example at the beginning of this manual.

\section{A.2.8 Other functions}

intrinsic HeckeBound ( $N$ :: RngIntElt, $k$ :: RngIntElt ) -> RngIntElt

intrinsic HeckeBound ( eps :: GrpDrchElt, $k$ :: RngIntElt ) -> RngIntEIt

These functions compute the Hecke bound for weight $\boldsymbol{k}$ and level $\mathbf{N}$, respectively Dirichelt character eps. Note that the Hecke bound is also often called the Sturm bound.

\section{A.3 Algebra handling}

\section{A.3.1 Affine algebras}

Let $A$ be a commutative local Artin algebra with maximal ideal $\mathfrak{m}$ over a finite field $k$. The residue field $K=A / \mathfrak{m}$ is a finite extension of $k$. By base changing to $K$ and taking one of the conjugate local factors, we now assume that $k=K$. The embedding dimension $e$ is the $k$-dimension of $\mathfrak{m} / \mathfrak{m}^{2}$. By Nakayama's Lemma, this is the minimal number of generators for $\mathfrak{m}$. The name comes from the fact that there is a surjection

$$
\pi: k\left[x_{1}, \ldots, x_{e}\right] \rightarrow A .
$$


Its kernel is called the relations ideal. By the nilpotency order we mean the maximal integer $n$ such that $m^{n}$ is not the zero ideal. (As the algebra is local and Artin, its maximal ideal is nilpotent.) We know that the ideal

$$
J^{n+1} \text { with } J:=\left(x_{1}, \ldots, x_{e}\right)
$$

is in the kernel of $\pi$. So, in order to store $\pi$, we only need to store the kernel $R$ of the linear map between two finite dimensional $k$-vector spaces

$$
\pi_{1}: k\left[x_{1}, \ldots, x_{e}\right] / J^{n+1} \rightarrow A .
$$

From the tuple $<k, e, n, R>$ the algebra can be recreated (up to isomorphism). Let us point out, however, that from the tuple it is not obvious whether two algebras are isomorphic. That would have to be tested after recreating the algebras.

These functions are used in order to store the Hecke algebras computed by HeckeAlgebras in a way that does not use much memory, but retains the algebra up to isomorphism.

intrinsic AffineAlgebra (A :: AlgMat : try_minimal := true) $->$ RngMPolRes intrinsic AffineAlgebra (A :: AlgAss : try_minimal := true) -> RngMPolRes

This function turns the local commutative algebra $\boldsymbol{A}$ into an affine algebra over its residue field. In fact, the algebra is first base changed to its residue field, then for one of the conjugate local factors an affine presentation is computed. If the option try_minimal is true, the number of relations will in general be smaller, but the computation time may be longer.

intrinsic AffineAlgebraTup (A :: AlgMat : try_minimal := true ) -> Tup intrinsic AffineAlgebraTup (A :: AlgAss : try_minimal := true) -> Tup

Given a commutative local Artin algebra $\boldsymbol{A}$, this function returns a tuple $\langle\mathbf{k}, \mathbf{e}, \mathbf{n}, \boldsymbol{R}\rangle$, consisting of the residue field $\mathbf{k}$ of $\boldsymbol{A}$, the embedding dimension $\mathbf{e}$, the nilpotency order $\boldsymbol{n}$ and relations $\boldsymbol{R}$. From these data, an affine algebra can be recreated which is isomorphic to one of the local factors of $A$ base changed to its residue field. If the option try_minimal is true, the number of relations will in general be smaller, but the computation time may be longer.

intrinsic AffineAlgebra (form :: Rec) -> RngMPolRes

Given a record of type AlgebraData, this function returns the corresponding Hecke algebra as an affine algebra.

intrinsic AffineAlgebra (A :: Tup) -> RngMPolRes

This function turns a tuple $\langle\mathbf{k}, \mathbf{e}, \mathbf{n}, \boldsymbol{R}\rangle$, as above consisting of a field $\mathbf{k}$, two integers $\mathbf{e}, \boldsymbol{n}$ (the embedding dimension and the nilpotency order) and relations $\boldsymbol{R}$, into an affine algebra.

\section{A.3.2 Matrix algebra functions}

intrinsic MatrixAlgebra ( $L$ :: SeqEnum ) -> AlgMat

Given a list of matrices $\boldsymbol{L}$, this function returns the matrix algebra generated by the members of $\boldsymbol{L}$.

intrinsic RegularRepresentation ( A :: AlgMat ) -> AlgMat

This function computes the regular representation of the commutative matrix algebra $\boldsymbol{A}$. 


\section{intrinsic CommonLowerTriangular ( A :: AlgMat ) -> AlgMat}

Given a local commutative matrix algebra $\boldsymbol{A}$, this function returns an isomorphic matrix algebra whose matrices are all lower triangular, after a scalar extension to the residue field and taking one of the Galois conjugate factors.

\section{Base change}

\section{intrinsic BaseChange ( $S$ :: Tup, T :: Tup ) -> Tup}

This function computes the composition of the base change matrices $\boldsymbol{T}=\langle\boldsymbol{C}, \boldsymbol{D}\rangle$, followed by those in $\boldsymbol{S}=\langle\boldsymbol{E}, \boldsymbol{F}\rangle$.

\section{intrinsic BaseChange ( $M$ :: Mtrx, T :: Tup ) -> Mtrx}

Given a matrix $\boldsymbol{M}$ and a tuple $\boldsymbol{T}=\langle\boldsymbol{C}, \boldsymbol{D}\rangle$ of base change matrices (for a subspace), this function computes the matrix of $\boldsymbol{M}$ with respect to the basis corresponding to $\boldsymbol{T}$.

\section{intrinsic BaseChange ( $M$ :: AlgMat, T :: Tup ) -> AlgMat}

Given a matrix algebra $\boldsymbol{M}$ and a tuple $\boldsymbol{T}=\langle\boldsymbol{C}, \boldsymbol{D}\rangle$ of base change matrices (for a subspace), this function computes the matrix algebra of $\boldsymbol{M}$ with respect to the basis corresponding to $\boldsymbol{T}$.

\section{Decomposition}

intrinsic Decomposition ( $M$ :: Mtrx : DegBound :=0 ) -> Tup intrinsic DecompositionUpToConjugation ( M :: Mtrx : DegBound :=0 ) -> Tup

Given a matrix $\boldsymbol{M}$, these functions compute a decomposition of the standard vector space such that $\boldsymbol{M}$ acts as multiplication by a scalar on each summand. The output is a tuple consisting of base change tuples $\langle\boldsymbol{C}, \boldsymbol{D}\rangle$ corresponding to the summands. With the second usage, summands conjugate under the absolute Galois group only appear once.

intrinsic Decomposition ( $L$ :: SeqEnum : DegBound :=0 ) -> Tup intrinsic DecompositionUpToConjugation ( $L$ :: SeqEnum : DegBound :=0) -> Tup Given a sequence $\boldsymbol{L}$ of commuting matrices, these functions compute a decomposition of the standard vector space such that each matrix in $\boldsymbol{L}$ acts as multiplication by a scalar on each summand. The output is a tuple consisting of base change tuples $\langle\boldsymbol{C , D}\rangle$ corresponding to the summands. With the second usage, summands conjugate under the absolute Galois group only appear once.

intrinsic Decomposition ( A :: AlgMat : DegBound :=0 ) -> Tup intrinsic DecompositionUpToConjugation ( A :: AlgMat : DegBound :=0 ) -> Tup Given a commutative matrix algebra $\boldsymbol{A}$, these functions compute a decomposition of the standard vector space such that each element in $\boldsymbol{A}$ acts as multiplication by a scalar on each summand. The output is a tuple consisting of base change tuples $\langle\boldsymbol{C}, \boldsymbol{D}\rangle$ corresponding to the summands. With the second usage, summands conjugate under the absolute Galois group only appear once.

intrinsic AlgebraDecomposition ( A :: AlgMat : DegBound :=0 ) -> SeqEnum intrinsic AlgebraDecompositionUpToConjugation ( A :: AlgMat : DegBound :=0 )

\section{-> SeqEnum}

Given a matrix algebra $\boldsymbol{A}$ over a finite field, these functions return a local factor of $\boldsymbol{A}$ after scalar extension to the residue field. With the second usage, factors conjugate under the absolute Galois group only appear once. 
intrinsic ChangeToResidueField ( A :: AlgMat ) -> SeqEnum

This function is identical to AlgebraDecompositionUpToConjugation.

\section{Localisations}

intrinsic Localisations ( $L$ :: SeqEnum ) -> Tup, Tup

intrinsic Localisations ( A :: AlgMat ) -> Tup, Tup

Given a list $\boldsymbol{L}$ of commuting matrices or a commutative matrix algebra $\boldsymbol{A}$, this function computes two tuples $\boldsymbol{C}, \boldsymbol{D}$, where $\boldsymbol{C}$ contains a tuple consisting of the localisations of $\boldsymbol{A}$, respectively of the matrix algebra generated by $\boldsymbol{L}$, and $\boldsymbol{D}$ consists of the corresponding base change tuples.

\section{A.3.3 Associative algebras}

\section{intrinsic Localisations ( A :: AlgAss ) -> SeqEnum}

This function returns a list of all localisations of the Artin algebra $\boldsymbol{A}$, which is assumed to be commutative. The output is a list of associative algebras.

\section{A.3.4 Gorenstein defect}

Let $A$ be a local Artin algebra over a field with unique maximal ideal $\mathfrak{m}$. We define the Gorenstein defect of $A$ to be $\left(\operatorname{dim}_{A / \mathfrak{m}} A[\mathfrak{m}]\right)-1$, which is equal to the number of $A$-module generators of the annihilator of the maximal ideal minus one. The algebra is said to be Gorenstein if its Gorenstein defect is equal to 0 .

intrinsic GorensteinDefect ( A :: RngMPolRes) -> RngIntEIt

intrinsic GorensteinDefect ( $A$ :: AlgAss) -> RngIntElt

intrinsic GorensteinDefect ( A :: AlgMat ) -> RngIntElt

These functions return the Gorenstein defect of the local commutative algebra $\boldsymbol{A}$.

intrinsic IsGorenstein ( $M$ :: RngMPolRes ) -> BoolElt

intrinsic IsGorenstein ( $M$ :: AlgAss ) -> BoolElt

intrinsic IsGorenstein ( $\mathrm{M}::$ AlgMat ) -> BoolElt

These functions test whether the commutative local algebra $\boldsymbol{M}$ is Gorenstein. 


\section{B Tables of Hecke algebras}

Characteristic $p=2$, prime levels, dihedral

\begin{tabular}{||c|c|c|c|c|c|c|c|c|c||}
\hline Level & Wt & ResD & Dim & EmbDim & NilO & GorDef & $\#$ Ops & $\#($ p $<$ HB $)$ & Gp \\
\hline $431 \frac{1}{2}$ & 2 & 1 & 4 & 3 & 1 & 2 & 6 & 20 & $D_{3}$ \\
5032 & 2 & 1 & 4 & 3 & 1 & 2 & 3 & 23 & $D_{3}$ \\
1319 & 2 & 2 & 4 & 3 & 1 & 2 & 6 & 47 & $D_{5}$ \\
1439 & 2 & 1 & 4 & 3 & 1 & 2 & 4 & 52 & $D_{3}$ \\
1559 & 2 & 1 & 4 & 3 & 1 & 2 & 7 & 55 & $D_{3}$ \\
1607 & 2 & 1 & 4 & 3 & 1 & 2 & 3 & 56 & $D_{3}$ \\
1759 & 2 & 1 & 4 & 3 & 1 & 2 & 5 & 62 & $D_{3}$ \\
1823 & 2 & 2 & 4 & 3 & 1 & 2 & 3 & 62 & $D_{5}$ \\
1879 & 2 & 1 & 16 & 4 & 5 & 2 & 6 & 65 & $D_{3}$ \\
1951 & 2 & 1 & 4 & 3 & 1 & 2 & 4 & 66 & $D_{3}$ \\
1999 & 2 & 1 & 4 & 3 & 1 & 2 & 5 & 67 & $D_{3}$ \\
2039 & 2 & 2 & 6 & 3 & 2 & 2 & 4 & 68 & $D_{5}$ \\
20893 & 2 & 1 & 18 & 4 & 7 & 2 & 5 & 70 & $D_{3}$ \\
2351 & 2 & 1 & 6 & 3 & 2 & 2 & 6 & 77 & $D_{3}$ \\
3407 & 2 & 1 & 16 & 4 & 5 & 2 & 7 & 103 & $D_{3}$ \\
3527 & 2 & 2 & 4 & 3 & 1 & 2 & 3 & 107 & $D_{5}$ \\
3623 & 2 & 1 & 6 & 3 & 2 & 2 & 3 & 110 & $D_{3}$ \\
3967 & 2 & 1 & 14 & 4 & 4 & 2 & 4 & 121 & $D_{3}$ \\
4231 & 2 & 1 & 4 & 3 & 1 & 2 & 10 & 126 & $D_{3}$ \\
4481 & 2 & 1 & 8 & 4 & 2 & 2 & 7 & 132 & $D_{3}$ \\
4799 & 2 & 1 & 12 & 4 & 3 & 2 & 5 & 139 & $D_{3}$ \\
4943 & 2 & 2 & 6 & 3 & 2 & 2 & 4 & 143 & $D_{5}$ \\
5167 & 2 & 1 & 6 & 3 & 2 & 2 & 5 & 149 & $D_{3}$ \\
5591 & 2 & 1 & 12 & 4 & 3 & 2 & 8 & 158 & $D_{3}$ \\
5591 & 2 & 3 & 4 & 3 & 1 & 2 & 5 & 158 & $D_{9}$ \\
5791 & 2 & 1 & 8 & 3 & 3 & 2 & 8 & 162 & $D_{3}$ \\
6199 & 2 & 1 & 16 & 4 & 5 & 2 & 7 & 174 & $D_{3}$ \\
6287 & 2 & 1 & 6 & 3 & 2 & 2 & 4 & 175 & $D_{3}$ \\
6343 & 2 & 1 & 12 & 4 & 3 & 2 & 5 & 177 & $D_{3}$ \\
6551 & 2 & 1 & 6 & 3 & 2 & 2 & 7 & 182 & $D_{3}$ \\
6823 & 2 & 1 & 4 & 3 & 1 & 2 & 4 & 189 & $D_{3}$ \\
6911 & 2 & 1 & 4 & 3 & 1 & 2 & 4 & 190 & $D_{3}$ \\
6967 & 2 & 1 & 12 & 4 & 3 & 2 & 8 & 191 & $D_{3}$ \\
7057 & 2 & 1 & 16 & 4 & 4 & 2 & 6 & 193 & $D_{3}$ \\
7103 & 2 & 3 & 4 & 3 & 1 & 2 & 3 & 194 & $D_{7}$ \\
7151 & 2 & 2 & 4 & 3 & 1 & 2 & 4 & 195 & $D_{5}$ \\
\hline
\end{tabular}

${ }^{1}$ See [14].

${ }^{2}$ See [14].

${ }^{3}$ See [14]. 


\begin{tabular}{|c|c|c|c|c|c|c|c|c|c|}
\hline Level & $\mathrm{Wt}$ & ResD & Dim & EmbDim & $\mathrm{NilO}$ & GorDef & \#Ops & $\#(\mathrm{p}<\mathrm{HB})$ & Gp \\
\hline 7351 & 2 & 1 & 12 & 4 & 3 & 2 & 9 & 200 & $\overline{D_{3}}$ \\
\hline 7487 & 2 & 2 & 6 & 3 & 2 & 2 & 3 & 203 & $D_{5}$ \\
\hline 7583 & 2 & 1 & 4 & 3 & 1 & 2 & 6 & 205 & $D_{3}$ \\
\hline 7951 & 2 & 1 & 4 & 3 & 1 & 2 & 6 & 216 & $D_{3}$ \\
\hline 8111 & 2 & 5 & 4 & 3 & 1 & 2 & 5 & 217 & $D_{11}$ \\
\hline 8167 & 2 & 1 & 6 & 3 & 2 & 2 & 4 & 218 & $D_{3}$ \\
\hline 8191 & 2 & 2 & 6 & 3 & 2 & 2 & 5 & 218 & $D_{5}$ \\
\hline 8623 & 2 & 1 & 4 & 3 & 1 & 2 & 8 & 227 & $D_{3}$ \\
\hline 8713 & 2 & 1 & 16 & 4 & 4 & 2 & 4 & 231 & $D_{3}$ \\
\hline 9127 & 2 & 1 & 16 & 4 & 5 & 2 & 4 & 240 & $D_{3}$ \\
\hline 9281 & 2 & 1 & 12 & 4 & 3 & 2 & 8 & 243 & $D_{3}$ \\
\hline 9439 & 2 & 1 & 8 & 3 & 3 & 2 & 4 & 248 & $D_{3}$ \\
\hline 9623 & 2 & 2 & 6 & 3 & 2 & 2 & 5 & 252 & $D_{5}$ \\
\hline 9967 & 2 & 1 & 6 & 3 & 2 & 2 & 4 & 260 & $D_{3}$ \\
\hline 10079 & 2 & 1 & 12 & 4 & 3 & 2 & 8 & 263 & $D_{3}$ \\
\hline 10103 & 2 & 1 & 4 & 3 & 1 & 2 & 4 & 263 & $D_{3}$ \\
\hline 10391 & 2 & 1 & 6 & 3 & 2 & 2 & 9 & 269 & $D_{3}$ \\
\hline 10391 & 2 & 3 & 4 & 3 & 1 & 2 & 8 & 269 & $D_{9}$ \\
\hline 10487 & 2 & 1 & 10 & 3 & 4 & 2 & 3 & 272 & $D_{3}$ \\
\hline 10567 & 2 & 1 & 12 & 3 & 5 & 2 & 5 & 274 & $D_{3}$ \\
\hline 10639 & 2 & 1 & 4 & 3 & 1 & 2 & 4 & 274 & $D_{3}$ \\
\hline 10663 & 2 & 1 & 6 & 3 & 2 & 2 & 9 & 275 & $D_{3}$ \\
\hline 10687 & 2 & 1 & 6 & 3 & 2 & 2 & 4 & 275 & $D_{3}$ \\
\hline 10799 & 2 & 1 & 14 & 3 & 6 & 2 & 8 & 278 & $D_{3}$ \\
\hline 11159 & 2 & 3 & 4 & 3 & 1 & 2 & 4 & 283 & $D_{7}$ \\
\hline 11321 & 2 & 1 & 8 & 4 & 2 & 2 & 9 & 289 & $D_{3}$ \\
\hline 11743 & 2 & 1 & 4 & 3 & 1 & 2 & 5 & 297 & $D_{3}$ \\
\hline 13063 & 2 & 1 & 6 & 3 & 2 & 2 & 5 & 326 & $D_{3}$ \\
\hline 13487 & 2 & 1 & 8 & 3 & 3 & 2 & 6 & 334 & $D_{3}$ \\
\hline 13999 & 2 & 3 & 4 & 3 & 1 & 2 & 4 & 345 & $D_{7}$ \\
\hline 14303 & 2 & 1 & 4 & 3 & 1 & 2 & 5 & 354 & $D_{3}$ \\
\hline 14543 & 2 & 3 & 4 & 3 & 1 & 2 & 3 & 360 & $D_{7}$ \\
\hline 14639 & 2 & 2 & 4 & 3 & 1 & 2 & 5 & 361 & $D_{5}$ \\
\hline 15121 & 2 & 2 & 8 & 4 & 2 & 2 & 6 & 369 & $D_{5}$ \\
\hline 15193 & 2 & 1 & 16 & 4 & 6 & 2 & 4 & 370 & $D_{3}$ \\
\hline 15271 & 2 & 1 & 6 & 3 & 2 & 2 & 8 & 372 & $D_{3}$ \\
\hline 15383 & 2 & 2 & 6 & 3 & 2 & 2 & 3 & 375 & $D_{5}$ \\
\hline 15391 & 2 & 1 & 6 & 3 & 2 & 2 & 7 & 375 & $D_{3}$ \\
\hline 15551 & 2 & 1 & 4 & 3 & 1 & 2 & 11 & 377 & $D_{3}$ \\
\hline 15607 & 2 & 1 & 6 & 3 & 2 & 2 & 8 & 378 & $D_{3}$ \\
\hline 15641 & 2 & 1 & 32 & 4 & 7 & 2 & 8 & 378 & $D_{3}$ \\
\hline 15919 & 2 & 1 & 26 & 4 & 7 & 2 & 6 & 383 & $D_{3}$ \\
\hline 15991 & 2 & 1 & 12 & 4 & 3 & 2 & 9 & 386 & $D_{3}$ \\
\hline
\end{tabular}




\begin{tabular}{||c|c|c|c|c|c|c|c|c|c||}
\hline Level & Wt & ResD & Dim & EmbDim & NilO & GorDef & $\#$ Ops & $\#(\mathrm{p}<$ HB $)$ & Gp \\
\hline 16127 & 2 & 3 & 4 & 3 & 1 & 2 & 3 & 390 & $D_{7}$ \\
16369 & 2 & 1 & 24 & 4 & 6 & 2 & 6 & 398 & $D_{3}$ \\
16487 & 2 & 1 & 6 & 3 & 2 & 2 & 4 & 400 & $D_{3}$ \\
16649 & 2 & 1 & 16 & 4 & 4 & 2 & 8 & 403 & $D_{3}$ \\
17471 & 2 & 1 & 6 & 3 & 2 & 2 & 11 & 421 & $D_{3}$ \\
18047 & 2 & 1 & 30 & 4 & 6 & 2 & 4 & 431 & $D_{3}$ \\
18097 & 2 & 1 & 36 & 5 & 6 & 2 & 9 & 432 & $D_{3}$ \\
18127 & 2 & 1 & 12 & 4 & 3 & 2 & 5 & 433 & $D_{3}$ \\
18257 & 2 & 1 & 12 & 4 & 4 & 2 & 4 & 436 & $D_{3}$ \\
19079 & 2 & 1 & 4 & 3 & 1 & 2 & 4 & 449 & $D_{3}$ \\
19079 & 2 & 3 & 4 & 3 & 1 & 2 & 4 & 449 & $D_{9}$ \\
19441 & 2 & 1 & 16 & 4 & 4 & 2 & 7 & 457 & $D_{3}$ \\
19543 & 2 & 2 & 4 & 3 & 1 & 2 & 4 & 460 & $D_{5}$ \\
19583 & 2 & 1 & 4 & 3 & 1 & 2 & 7 & 461 & $D_{3}$ \\
19751 & 2 & 1 & 4 & 3 & 1 & 2 & 4 & 462 & $D_{3}$ \\
19919 & 2 & 1 & 16 & 4 & 5 & 2 & 7 & 467 & $D_{3}$ \\
19927 & 2 & 1 & 6 & 3 & 2 & 2 & 4 & 467 & $D_{3}$ \\
20183 & 2 & 2 & 4 & 3 & 1 & 2 & 7 & 474 & $D_{5}$ \\
20599 & 2 & 1 & 6 & 3 & 2 & 2 & 6 & 481 & $D_{3}$ \\
20759 & 2 & 1 & 18 & 4 & 6 & 2 & 9 & 483 & $D_{3}$ \\
20887 & 2 & 2 & 6 & 3 & 2 & 2 & 4 & 487 & $D_{5}$ \\
21319 & 2 & 3 & 4 & 3 & 1 & 2 & 9 & 497 & $D_{7}$ \\
21647 & 2 & 1 & 6 & 3 & 2 & 2 & 6 & 504 & $D_{3}$ \\
21737 & 2 & 1 & 24 & 5 & 4 & 2 & 7 & 507 & $D_{3}$ \\
21839 & 2 & 2 & 4 & 3 & 1 & 2 & 7 & 509 & $D_{5}$ \\
22159 & 2 & 2 & 4 & 3 & 1 & 2 & 9 & 515 & $D_{5}$ \\
22511 & 2 & 1 & 4 & 3 & 1 & 2 & 6 & 522 & $D_{3}$ \\
22567 & 2 & 3 & 4 & 3 & 1 & 2 & 4 & 523 & $D_{7}$ \\
22751 & 2 & 3 & 4 & 3 & 1 & 2 & 4 & 526 & $D_{7}$ \\
23159 & 2 & 1 & 20 & 4 & 6 & 2 & 6 & 535 & $D_{3}$ \\
23159 & 2 & 3 & 6 & 3 & 2 & 2 & 5 & 535 & $D_{9}$ \\
23279 & 2 & 1 & 8 & 3 & 3 & 2 & 4 & 537 & $D_{3}$ \\
23321 & 2 & 1 & 12 & 4 & 4 & 2 & 7 & 538 & $D_{3}$ \\
23417 & 2 & 1 & 26 & 4 & 7 & 2 & 10 & 539 & $D_{3}$ \\
23567 & 2 & 1 & 4 & 3 & 1 & 2 & 3 & 544 & $D_{3}$ \\
23687 & 2 & 3 & 4 & 3 & 1 & 2 & 3 & 548 & $D_{7}$ \\
23743 & 2 & 2 & 4 & 3 & 1 & 2 & 4 & 548 & $D_{5}$ \\
24151 & 2 & 2 & 4 & 3 & 1 & 2 & 5 & 556 & $D_{5}$ \\
24281 & 2 & 1 & 16 & 4 & 4 & 2 & 5 & 557 & $D_{3}$ \\
24439 & 2 & 2 & 6 & 3 & 2 & 2 & 7 & 561 & $D_{5}$ \\
24847 & 2 & 2 & 4 & 3 & 1 & 2 & 8 & 570 & $D_{5}$ \\
25031 & 2 & 1 & 8 & 3 & 3 & 2 & 7 & 573 & $D_{3}$ \\
25111 & 2 & 1 & 6 & 3 & 2 & 2 & 8 & 574 & $D_{3}$ \\
\hline
\end{tabular}




\begin{tabular}{|c|c|c|c|c|c|c|c|c|c|}
\hline Level & $\mathrm{Wt}$ & ResD & Dim & EmbDim & $\mathrm{NilO}$ & GorDef & \#Ops & $\#(\mathrm{p}<\mathrm{HB})$ & Gp \\
\hline 25247 & 2 & 1 & 6 & 3 & 2 & 2 & 10 & 575 & $D_{3}$ \\
\hline 25409 & 2 & 1 & 8 & 4 & 2 & 2 & 8 & 580 & $D_{3}$ \\
\hline 25439 & 2 & 1 & 4 & 3 & 1 & 2 & 6 & 580 & $D_{3}$ \\
\hline 25447 & 2 & 1 & 6 & 3 & 2 & 2 & 11 & 581 & $D_{3}$ \\
\hline 25793 & 2 & 1 & 16 & 4 & 4 & 2 & 5 & 590 & $D_{3}$ \\
\hline 26431 & 2 & 2 & 6 & 3 & 2 & 2 & 4 & 599 & $D_{5}$ \\
\hline 26839 & 2 & 3 & 4 & 3 & 1 & 2 & 5 & 607 & $D_{7}$ \\
\hline 26959 & 2 & 1 & 6 & 3 & 2 & 2 & 23 & 610 & $D_{3}$ \\
\hline 27143 & 2 & 1 & 4 & 3 & 1 & 2 & 4 & 615 & $D_{3}$ \\
\hline 27143 & 2 & 3 & 4 & 3 & 1 & 2 & 3 & 615 & $D_{9}$ \\
\hline 27647 & 2 & 1 & 26 & 4 & 10 & 2 & 7 & 623 & $D_{3}$ \\
\hline 27673 & 2 & 1 & 8 & 4 & 2 & 2 & 7 & 623 & $D_{3}$ \\
\hline 27743 & 2 & 3 & 4 & 3 & 1 & 2 & 3 & 624 & $D_{7}$ \\
\hline 28031 & 2 & 2 & 6 & 3 & 2 & 2 & 5 & 631 & $D_{5}$ \\
\hline 28031 & 2 & 1 & 8 & 3 & 3 & 2 & 5 & 631 & $D_{3}$ \\
\hline 28031 & 2 & 4 & 4 & 3 & 1 & 2 & 7 & 631 & $D_{15}$ \\
\hline 28279 & 2 & 1 & 8 & 3 & 3 & 2 & 4 & 635 & $D_{3}$ \\
\hline 28279 & 2 & 1 & 26 & 4 & 8 & 2 & 14 & 635 & $D_{3}$ \\
\hline 28279 & 2 & 1 & 4 & 3 & 1 & 2 & 11 & 635 & $D_{3}$ \\
\hline 28279 & 2 & 1 & 6 & 3 & 2 & 2 & 6 & 635 & $D_{3}$ \\
\hline 28703 & 2 & 1 & 20 & 4 & 5 & 2 & 5 & 642 & $D_{3}$ \\
\hline 28759 & 2 & 1 & 8 & 3 & 3 & 2 & 5 & 645 & $D_{3}$ \\
\hline 29023 & 2 & 1 & 4 & 3 & 1 & 2 & 8 & 650 & $D_{3}$ \\
\hline 29287 & 2 & 2 & 8 & 3 & 3 & 2 & 4 & 653 & $D_{5}$ \\
\hline 29311 & 2 & 3 & 6 & 3 & 2 & 2 & 5 & 653 & $D_{7}$ \\
\hline 29399 & 2 & 1 & 6 & 3 & 2 & 2 & 7 & 654 & $D_{3}$ \\
\hline 29567 & 2 & 1 & 6 & 3 & 2 & 2 & 6 & 657 & $D_{3}$ \\
\hline 29879 & 2 & 2 & 4 & 3 & 1 & 2 & 5 & 666 & $D_{5}$ \\
\hline 29959 & 2 & 1 & 6 & 3 & 2 & 2 & 4 & 668 & $D_{3}$ \\
\hline 29959 & 2 & 3 & 4 & 3 & 1 & 2 & 4 & 668 & $D_{9}$ \\
\hline 30223 & 2 & 1 & 8 & 3 & 3 & 2 & 5 & 674 & $D_{3}$ \\
\hline 30367 & 2 & 2 & 6 & 3 & 2 & 2 & 5 & 677 & $D_{5}$ \\
\hline 30431 & 2 & 2 & 4 & 3 & 1 & 2 & 4 & 677 & $D_{5}$ \\
\hline 30559 & 2 & 3 & 4 & 3 & 1 & 2 & 9 & 680 & $D_{7}$ \\
\hline 30727 & 2 & 1 & 6 & 3 & 2 & 2 & 9 & 685 & $D_{3}$ \\
\hline 30911 & 2 & 2 & 4 & 3 & 1 & 2 & 5 & 686 & $D_{5}$ \\
\hline 31079 & 2 & 2 & 4 & 3 & 1 & 2 & 5 & 690 & $D_{5}$ \\
\hline 31159 & 2 & 1 & 16 & 4 & 5 & 2 & 6 & 691 & $D_{3}$ \\
\hline 31247 & 2 & 6 & 4 & 3 & 1 & 2 & 3 & 692 & $D_{13}$ \\
\hline 31271 & 2 & 1 & 6 & 3 & 2 & 2 & 9 & 693 & $D_{3}$ \\
\hline 31321 & 2 & 3 & 8 & 4 & 2 & 2 & 7 & 693 & $D_{7}$ \\
\hline 31513 & 2 & 1 & 16 & 4 & 4 & 2 & 4 & 697 & $D_{3}$ \\
\hline 31543 & 2 & 2 & 4 & 3 & 1 & 2 & 5 & 697 & $D_{5}$ \\
\hline
\end{tabular}




\begin{tabular}{|c|c|c|c|c|c|c|c|c|c|}
\hline Level & $\mathrm{Wt}$ & ResD & Dim & EmbDim & $\mathrm{NilO}$ & GorDef & \#Ops & $\#(\mathrm{p}<\mathrm{HB})$ & $\mathrm{Gp}$ \\
\hline 31847 & 2 & 5 & 4 & 3 & 1 & 2 & 3 & 703 & $D_{11}$ \\
\hline 32009 & 2 & 1 & 12 & 4 & 3 & 2 & 9 & 706 & $D_{3}$ \\
\hline 32143 & 2 & 3 & 4 & 3 & 1 & 2 & 4 & 708 & $D_{7}$ \\
\hline 32183 & 2 & 3 & 4 & 3 & 1 & 2 & 3 & 708 & $D_{7}$ \\
\hline 32327 & 2 & 1 & 16 & 4 & 5 & 2 & 5 & 710 & $D_{3}$ \\
\hline 32327 & 2 & 3 & 4 & 3 & 1 & 2 & 3 & 710 & $D_{9}$ \\
\hline 32353 & 2 & 1 & 20 & 4 & 6 & 2 & 5 & 711 & $D_{3}$ \\
\hline 32401 & 2 & 1 & 20 & 4 & 6 & 2 & 6 & 712 & $D_{3}$ \\
\hline 32479 & 2 & 5 & 4 & 3 & 1 & 2 & 4 & 714 & $D_{11}$ \\
\hline 32647 & 2 & 3 & 4 & 3 & 1 & 2 & 4 & 719 & $D_{7}$ \\
\hline 32687 & 2 & 5 & 4 & 3 & 1 & 2 & 3 & 720 & $D_{11}$ \\
\hline 32719 & 2 & 2 & 4 & 3 & 1 & 2 & 14 & 721 & $D_{5}$ \\
\hline 32887 & 2 & 2 & 6 & 3 & 2 & 2 & 6 & 724 & $D_{5}$ \\
\hline 32983 & 2 & 1 & 4 & 3 & 1 & 2 & 4 & 725 & $D_{3}$ \\
\hline 33223 & 2 & 1 & 4 & 3 & 1 & 2 & 9 & 732 & $D_{3}$ \\
\hline 33343 & 2 & 1 & 4 & 3 & 1 & 2 & 5 & 733 & $D_{3}$ \\
\hline 33679 & 2 & 1 & 4 & 3 & 1 & 2 & 5 & 738 & $D_{3}$ \\
\hline 33767 & 2 & 3 & 4 & 3 & 1 & 2 & 3 & 739 & $D_{7}$ \\
\hline 34351 & 2 & 2 & 4 & 3 & 1 & 2 & 8 & 753 & $D_{5}$ \\
\hline 34471 & 2 & 2 & 12 & 4 & 3 & 2 & 6 & 756 & $D_{5}$ \\
\hline 34487 & 2 & 1 & 14 & 4 & 4 & 2 & 7 & 756 & $D_{3}$ \\
\hline 34591 & 2 & 2 & 4 & 3 & 1 & 2 & 5 & 757 & $D_{5}$ \\
\hline 34679 & 2 & 1 & 6 & 3 & 2 & 2 & 4 & 758 & $D_{3}$ \\
\hline 34679 & 2 & 3 & 4 & 3 & 1 & 2 & 4 & 758 & $D_{9}$ \\
\hline 34721 & 2 & 1 & 12 & 4 & 4 & 2 & 9 & 759 & $D_{3}$ \\
\hline 34847 & 2 & 1 & 16 & 4 & 5 & 2 & 6 & 762 & $D_{3}$ \\
\hline 35401 & 2 & 1 & 56 & 5 & 8 & 2 & 10 & 776 & $D_{3}$ \\
\hline 35591 & 2 & 9 & 4 & 3 & 1 & 2 & 7 & 779 & $D_{19}$ \\
\hline 35759 & 2 & 3 & 6 & 3 & 2 & 2 & 5 & 781 & $D_{7}$ \\
\hline 35839 & 2 & 2 & 4 & 3 & 1 & 2 & 5 & 781 & $D_{5}$ \\
\hline 35977 & 2 & 2 & 8 & 4 & 2 & 2 & 5 & 783 & $D_{5}$ \\
\hline 36191 & 2 & 1 & 46 & 5 & 7 & 2 & 11 & 786 & $D_{3}$ \\
\hline 36791 & 2 & 1 & 12 & 4 & 3 & 2 & 8 & 799 & $D_{3}$ \\
\hline 36871 & 2 & 2 & 6 & 3 & 2 & 2 & 5 & 801 & $D_{5}$ \\
\hline 37087 & 2 & 1 & 32 & 4 & 11 & 2 & 5 & 804 & $D_{3}$ \\
\hline 37199 & 2 & 1 & 18 & 4 & 4 & 2 & 8 & 806 & $D_{3}$ \\
\hline 37607 & 2 & 1 & 4 & 3 & 1 & 2 & 3 & 814 & $D_{3}$ \\
\hline 37831 & 2 & 2 & 4 & 3 & 1 & 2 & 5 & 820 & $D_{5}$ \\
\hline 37879 & 2 & 3 & 4 & 3 & 1 & 2 & 5 & 821 & $D_{7}$ \\
\hline 37993 & 2 & 2 & 8 & 4 & 2 & 2 & 5 & 824 & $D_{5}$ \\
\hline 38047 & 2 & 2 & 4 & 3 & 1 & 2 & 9 & 825 & $D_{5}$ \\
\hline 38167 & 2 & 1 & 14 & 4 & 4 & 2 & 10 & 829 & $D_{3}$ \\
\hline 38231 & 2 & 1 & 4 & 3 & 1 & 2 & 4 & 830 & $D_{3}$ \\
\hline
\end{tabular}




\begin{tabular}{||c|c|c|c|c|c|c|c|c|c||}
\hline Level & Wt & ResD & Dim & EmbDim & NilO & GorDef & $\#$ Ops & $\#(\mathrm{p}<\mathrm{HB})$ & $\mathrm{Gp}$ \\
\hline 38287 & 2 & 1 & 4 & 3 & 1 & 2 & 5 & 832 & $D_{3}$ \\
38303 & 2 & 1 & 4 & 3 & 1 & 2 & 3 & 832 & $D_{3}$ \\
38593 & 2 & 1 & 20 & 4 & 6 & 2 & 9 & 836 & $D_{3}$ \\
38959 & 2 & 1 & 10 & 3 & 4 & 2 & 9 & 842 & $D_{3}$ \\
38977 & 2 & 1 & 20 & 4 & 6 & 2 & 13 & 842 & $D_{3}$ \\
39023 & 2 & 1 & 40 & 5 & 7 & 2 & 10 & 842 & $D_{3}$ \\
39199 & 2 & 2 & 4 & 3 & 1 & 2 & 7 & 844 & $D_{5}$ \\
39631 & 2 & 1 & 16 & 4 & 5 & 2 & 11 & 853 & $D_{3}$ \\
39679 & 2 & 1 & 6 & 3 & 2 & 2 & 12 & 854 & $D_{3}$ \\
39679 & 2 & 3 & 4 & 3 & 1 & 2 & 4 & 854 & $D_{9}$ \\
\hline \hline
\end{tabular}

Characteristic $p=2$ and non-prime square-free levels, dihedral

\begin{tabular}{||c|c|c|c|c|c|c|c|c|c||}
\hline Level & Wt & ResD & Dim & EmbDim & NilO & GorDef & \#Ops & $\#(\mathrm{p}<\mathrm{HB})$ & $\mathrm{Gp}$ \\
\hline 1055 & 2 & 1 & 24 & 4 & 9 & 2 & 5 & 47 & $D_{3}$ \\
1727 & 2 & 1 & 16 & 4 & 5 & 2 & 6 & 65 & $D_{3}$ \\
20714 & 2 & 1 & 8 & 4 & 2 & 2 & 5 & 73 & $D_{3}$ \\
2631 & 2 & 1 & 40 & 4 & 17 & 2 & 6 & 106 & $D_{3}$ \\
2991 & 2 & 1 & 40 & 4 & 17 & 2 & 4 & 121 & $D_{3}$ \\
3095 & 2 & 1 & 40 & 4 & 17 & 2 & 4 & 114 & $D_{3}$ \\
3431 & 2 & 1 & 24 & 5 & 4 & 2 & 6 & 107 & $D_{3}$ \\
3471 & 2 & 1 & 16 & 5 & 3 & 2 & 5 & 146 & $D_{3}$ \\
3639 & 2 & 1 & 28 & 4 & 11 & 2 & 5 & 140 & $D_{3}$ \\
4031 & 2 & 1 & 16 & 4 & 4 & 2 & 6 & 125 & $D_{3}$ \\
4087 & 2 & 1 & 8 & 4 & 2 & 2 & 6 & 126 & $D_{3}$ \\
4119 & 2 & 1 & 12 & 4 & 3 & 2 & 4 & 156 & $D_{3}$ \\
4415 & 2 & 1 & 8 & 4 & 2 & 2 & 6 & 153 & $D_{3}$ \\
\hline \hline
\end{tabular}

Characteristic $p=2$, icosahedral

\begin{tabular}{||c|c|c|c|c|c|c|c|c|c||}
\hline Level & Wt & ResD & Dim & EmbDim & NilO & GorDef & $\#$ Ops & $\#(\mathrm{p}<\mathrm{HB})$ & Gp \\
\hline 89491 & 2 & 2 & 12 & 4 & 3 & 2 & 4 & 1746 & $A_{5}$ \\
\hline \hline
\end{tabular}

Characteristic $p=3$, prime levels, dihedral

\begin{tabular}{||c|c|c|c|c|c|c|c|c|c||}
\hline Level & Wt & ResD & Dim & EmbDim & NilO & GorDef & \#Ops & \#(p $<$ HB $)$ & Gp \\
\hline 1031 & 3 & 2 & 4 & 3 & 1 & 2 & 4 & 55 & $D_{5}$ \\
1511 & 3 & 3 & 4 & 3 & 1 & 2 & 9 & 74 & $D_{7}$ \\
2087 & 3 & 2 & 4 & 3 & 1 & 2 & 3 & 98 & $D_{5}$ \\
\hline
\end{tabular}

\footnotetext{
${ }^{4}$ First found by W. Stein.
} 


\begin{tabular}{||c|c|c|c|c|c|c|c|c|c||}
\hline Level & Wt & ResD & Dim & EmbDim & NilO & GorDef & \#Ops & $\#(\mathrm{p}<$ HB $)$ & Gp \\
\hline 4259 & 3 & 2 & 4 & 3 & 1 & 2 & 3 & 179 & $D_{5}$ \\
4799 & 3 & 3 & 22 & 4 & 9 & 2 & 9 & 196 & $D_{7}$ \\
5939 & 3 & 2 & 4 & 3 & 1 & 2 & 4 & 235 & $D_{5}$ \\
6899 & 3 & 2 & 4 & 3 & 1 & 2 & 3 & 269 & $D_{5}$ \\
6959 & 3 & 2 & 4 & 3 & 1 & 2 & 4 & 270 & $D_{5}$ \\
7523 & 3 & 2 & 4 & 3 & 1 & 2 & 4 & 289 & $D_{5}$ \\
7559 & 3 & 2 & 4 & 3 & 1 & 2 & 6 & 290 & $D_{5}$ \\
7583 & 3 & 3 & 20 & 3 & 9 & 2 & 6 & 290 & $D_{7}$ \\
8219 & 3 & 2 & 4 & 3 & 1 & 2 & 6 & 310 & $D_{5}$ \\
8447 & 3 & 5 & 20 & 3 & 9 & 2 & 3 & 318 & $D_{11}$ \\
8699 & 3 & 2 & 6 & 3 & 2 & 2 & 9 & 326 & $D_{5}$ \\
9431 & 3 & 3 & 4 & 3 & 1 & 2 & 4 & 350 & $D_{7}$ \\
9743 & 3 & 2 & 8 & 3 & 3 & 2 & 3 & 360 & $D_{5}$ \\
9887 & 3 & 2 & 8 & 3 & 3 & 2 & 3 & 365 & $D_{5}$ \\
10079 & 3 & 2 & 60 & 3 & 29 & 2 & 5 & 368 & $D_{5}$ \\
10247 & 3 & 2 & 10 & 4 & 3 & 2 & 5 & 375 & $D_{5}$ \\
10847 & 3 & 3 & 22 & 4 & 9 & 2 & 9 & 395 & $D_{7}$ \\
12011 & 3 & 2 & 4 & 3 & 1 & 2 & 3 & 431 & $D_{5}$ \\
12119 & 3 & 2 & 56 & 3 & 27 & 2 & 8 & 434 & $D_{5}$ \\
12263 & 3 & 2 & 8 & 3 & 3 & 2 & 3 & 438 & $D_{5}$ \\
12959 & 3 & 5 & 20 & 3 & 9 & 2 & 4 & 457 & $D_{11}$ \\
13907 & 3 & 2 & 22 & 4 & 9 & 2 & 8 & 487 & $D_{5}$ \\
14699 & 3 & 2 & 4 & 3 & 1 & 2 & 6 & 513 & $D_{5}$ \\
14783 & 3 & 3 & 20 & 3 & 9 & 2 & 3 & 515 & $D_{13}$ \\
14783 & 3 & 3 & 20 & 3 & 9 & 2 & 3 & 515 & $D_{13}$ \\
\hline \hline
\end{tabular}

Characteristic $p=5$, prime levels, dihedral

\begin{tabular}{||c|c|c|c|c|c|c|c|c|c||}
\hline Level & Wt & ResD & Dim & EmbDim & NilO & GorDef & \#Ops & $\#(\mathrm{p}<\mathrm{HB})$ & $\mathrm{Gp}$ \\
\hline 419 & 5 & 1 & 4 & 3 & 1 & 2 & 3 & 40 & $D_{3}$ \\
439 & 5 & 1 & 14 & 4 & 5 & 2 & 10 & 42 & $D_{3}$ \\
491 & 5 & 1 & 4 & 3 & 1 & 2 & 3 & 46 & $D_{3}$ \\
751 & 5 & 1 & 12 & 3 & 5 & 2 & 3 & 65 & $D_{3}$ \\
839 & 5 & 1 & 6 & 3 & 2 & 2 & 6 & 70 & $D_{3}$ \\
1231 & 5 & 1 & 4 & 3 & 1 & 2 & 3 & 97 & $D_{3}$ \\
2579 & 5 & 1 & 4 & 3 & 1 & 2 & 3 & 180 & $D_{3}$ \\
2699 & 5 & 1 & 14 & 4 & 5 & 2 & 8 & 188 & $D_{3}$ \\
3299 & 5 & 1 & 4 & 3 & 1 & 2 & 6 & 220 & $D_{3}$ \\
3359 & 5 & 1 & 4 & 3 & 1 & 2 & 6 & 222 & $D_{3}$ \\
4111 & 5 & 1 & 4 & 3 & 1 & 2 & 3 & 267 & $D_{3}$ \\
4219 & 5 & 1 & 20 & 3 & 6 & 2 & 5 & 274 & $D_{3}$ \\
4931 & 5 & 3 & 12 & 3 & 5 & 2 & 3 & 310 & $D_{7}$ \\
5011 & 5 & 1 & 4 & 3 & 1 & 2 & 5 & 316 & $D_{3}$ \\
\hline
\end{tabular}




\begin{tabular}{||c|c|c|c|c|c|c|c|c|c||}
\hline Level & Wt & ResD & Dim & EmbDim & NilO & GorDef & \#Ops & $\#(\mathrm{p}<\mathrm{HB})$ & Gp \\
\hline 5639 & 5 & 1 & 6 & 3 & 2 & 2 & 5 & 348 & $D_{3}$ \\
5939 & 5 & 3 & 12 & 3 & 5 & 2 & 5 & 366 & $D_{7}$ \\
6079 & 5 & 1 & 6 & 3 & 2 & 2 & 5 & 370 & $D_{3}$ \\
6271 & 5 & 1 & 4 & 3 & 1 & 2 & 3 & 379 & $D_{3}$ \\
6571 & 5 & 1 & 12 & 3 & 5 & 2 & 5 & 399 & $D_{3}$ \\
6691 & 5 & 1 & 4 & 3 & 1 & 2 & 5 & 405 & $D_{3}$ \\
6779 & 5 & 1 & 6 & 3 & 2 & 2 & 6 & 410 & $D_{3}$ \\
7459 & 5 & 1 & 12 & 3 & 5 & 2 & 7 & 443 & $D_{3}$ \\
7759 & 5 & 3 & 4 & 3 & 1 & 2 & 3 & 457 & $D_{7}$ \\
8779 & 5 & 1 & 12 & 3 & 5 & 2 & 12 & 511 & $D_{3}$ \\
8819 & 5 & 3 & 4 & 3 & 1 & 2 & 3 & 513 & $D_{7}$ \\
9011 & 5 & 1 & 4 & 3 & 1 & 2 & 6 & 522 & $D_{3}$ \\
\hline \hline
\end{tabular}

Characteristic $p=7$, prime levels, dihedral

\begin{tabular}{||c|c|c|c|c|c|c|c|c|c||}
\hline Level & Wt & ResD & Dim & EmbDim & NilO & GorDef & \#Ops & \#(p<HB $)$ & Gp \\
\hline 199 & 7 & 1 & 4 & 3 & 1 & 2 & 3 & 30 & $D_{3}$ \\
839 & 7 & 1 & 4 & 3 & 1 & 2 & 6 & 93 & $D_{3}$ \\
1259 & 7 & 1 & 4 & 3 & 1 & 2 & 3 & 130 & $D_{3}$ \\
1291 & 7 & 1 & 4 & 3 & 1 & 2 & 4 & 133 & $D_{3}$ \\
1319 & 7 & 1 & 4 & 3 & 1 & 2 & 6 & 136 & $D_{3}$ \\
1399 & 7 & 1 & 4 & 3 & 1 & 2 & 3 & 141 & $D_{3}$ \\
1559 & 7 & 1 & 4 & 3 & 1 & 2 & 7 & 155 & $D_{3}$ \\
1567 & 7 & 1 & 8 & 3 & 4 & 2 & 3 & 156 & $D_{3}$ \\
1823 & 7 & 1 & 6 & 3 & 2 & 2 & 4 & 179 & $D_{3}$ \\
1823 & 7 & 3 & 4 & 3 & 1 & 2 & 4 & 179 & $D_{9}$ \\
\hline \hline
\end{tabular}

Characteristic $p=11$, prime levels, dihedral

\begin{tabular}{||c|c|c|c|c|c|c|c|c|c||}
\hline Level & Wt & ResD & Dim & EmbDim & NilO & GorDef & \#Ops & $\#(\mathrm{p}<\mathrm{HB})$ & Gp \\
\hline 431 & 11 & 3 & 4 & 3 & 1 & 2 & 5 & 77 & $D_{7}$ \\
563 & 11 & 1 & 4 & 3 & 1 & 2 & 3 & 97 & $D_{3}$ \\
1187 & 11 & 1 & 6 & 3 & 2 & 2 & 6 & 181 & $D_{3}$ \\
1223 & 11 & 3 & 4 & 3 & 1 & 2 & 4 & 187 & $D_{7}$ \\
1231 & 11 & 1 & 4 & 3 & 1 & 2 & 3 & 189 & $D_{3}$ \\
1231 & 11 & 3 & 4 & 3 & 1 & 2 & 3 & 189 & $D_{9}$ \\
1327 & 11 & 1 & 4 & 3 & 1 & 2 & 3 & 199 & $D_{5}$ \\
1327 & 11 & 1 & 4 & 3 & 1 & 2 & 4 & 199 & $D_{5}$ \\
1583 & 11 & 1 & 24 & 3 & 11 & 2 & 4 & 230 & $D_{3}$ \\
1619 & 11 & 1 & 4 & 3 & 1 & 2 & 3 & 235 & $D_{3}$ \\
1823 & 11 & 1 & 4 & 3 & 1 & 2 & 4 & 263 & $D_{3}$ \\
2243 & 11 & 1 & 4 & 3 & 1 & 2 & 3 & 310 & $D_{3}$ \\
\hline
\end{tabular}




\begin{tabular}{||c|c|c|c|c|c|c|c|c|c||}
\hline Level & Wt & ResD & Dim & EmbDim & NilO & GorDef & \#Ops & $\#(\mathrm{p}<\mathrm{HB})$ & Gp \\
\hline 2351 & 11 & 1 & 4 & 3 & 1 & 2 & 6 & 325 & $D_{3}$ \\
2351 & 11 & 3 & 4 & 3 & 1 & 2 & 6 & 325 & $D_{9}$ \\
2503 & 11 & 1 & 4 & 3 & 1 & 2 & 3 & 341 & $D_{3}$ \\
2591 & 11 & 1 & 4 & 3 & 1 & 2 & 5 & 351 & $D_{3}$ \\
2647 & 11 & 1 & 4 & 3 & 1 & 2 & 3 & 360 & $D_{3}$ \\
2767 & 11 & 1 & 4 & 3 & 1 & 2 & 3 & 370 & $D_{3}$ \\
2791 & 11 & 1 & 4 & 3 & 1 & 2 & 3 & 375 & $D_{3}$ \\
3011 & 11 & 1 & 4 & 3 & 1 & 2 & 5 & 402 & $D_{3}$ \\
3119 & 11 & 1 & 4 & 3 & 1 & 2 & 5 & 415 & $D_{3}$ \\
3299 & 11 & 1 & 4 & 3 & 1 & 2 & 4 & 434 & $D_{3}$ \\
3299 & 11 & 3 & 4 & 3 & 1 & 2 & 4 & 434 & $D_{9}$ \\
3571 & 11 & 1 & 4 & 3 & 1 & 2 & 4 & 462 & $D_{3}$ \\
\hline \hline
\end{tabular}

Characteristic $p=13$, prime levels, dihedral

\begin{tabular}{||c|c|c|c|c|c|c|c|c|c||}
\hline Level & Wt & ResD & Dim & EmbDim & NilO & GorDef & \#Ops & \#(p<HB $)$ & Gp \\
\hline 367 & 13 & 1 & 4 & 3 & 1 & 2 & 3 & 78 & $D_{3}$ \\
439 & 13 & 2 & 4 & 3 & 1 & 2 & 4 & 91 & $D_{5}$ \\
563 & 13 & 1 & 4 & 3 & 1 & 2 & 3 & 111 & $D_{3}$ \\
971 & 13 & 2 & 4 & 3 & 1 & 2 & 4 & 177 & $D_{5}$ \\
1223 & 13 & 2 & 4 & 3 & 1 & 2 & 4 & 216 & $D_{5}$ \\
1427 & 13 & 1 & 4 & 3 & 1 & 2 & 5 & 243 & $D_{3}$ \\
1439 & 13 & 1 & 28 & 3 & 13 & 2 & 5 & 246 & $D_{3}$ \\
1823 & 13 & 1 & 4 & 3 & 1 & 2 & 4 & 298 & $D_{3}$ \\
\hline \hline
\end{tabular}

Characteristic $p=17$, prime levels, dihedral

\begin{tabular}{||c|c|c|c|c|c|c|c|c|c||}
\hline Level & Wt & ResD & Dim & EmbDim & NilO & GorDef & \#Ops & $\#(\mathrm{p}<\mathrm{HB})$ & Gp \\
\hline 59 & 17 & 1 & 6 & 3 & 2 & 2 & 3 & 23 & $D_{3}$ \\
239 & 17 & 2 & 4 & 3 & 1 & 2 & 5 & 68 & $D_{5}$ \\
1327 & 17 & 1 & 4 & 3 & 1 & 2 & 3 & 289 & $D_{3}$ \\
1427 & 17 & 2 & 4 & 3 & 1 & 2 & 3 & 306 & $D_{5}$ \\
1951 & 17 & 1 & 4 & 3 & 1 & 2 & 4 & 402 & $D_{3}$ \\
2503 & 17 & 1 & 4 & 3 & 1 & 2 & 3 & 497 & $D_{3}$ \\
2687 & 17 & 1 & 36 & 3 & 17 & 2 & 4 & 529 & $D_{3}$ \\
\hline \hline
\end{tabular}

Characteristic $p=19$, prime levels, dihedral

\begin{tabular}{||c|c|c|c|c|c|c|c|c|c||}
\hline Level & Wt & ResD & Dim & EmbDim & NilO & GorDef & \#Ops & \#(p $<$ HB $)$ & Gp \\
\hline 439 & 19 & 1 & 6 & 3 & 2 & 2 & 3 & 125 & $D_{3}$ \\
751 & 19 & 1 & 4 & 3 & 1 & 2 & 5 & 195 & $D_{5}$ \\
\hline
\end{tabular}




\begin{tabular}{||c|c|c|c|c|c|c|c|c|c||}
\hline Level & Wt & ResD & Dim & EmbDim & NilO & GorDef & \#Ops & $\#(\mathrm{p}<$ HB $)$ & Gp \\
\hline 751 & 19 & 1 & 4 & 3 & 1 & 2 & 5 & 195 & $D_{5}$ \\
1427 & 19 & 1 & 6 & 3 & 2 & 2 & 3 & 335 & $D_{3}$ \\
\hline \hline
\end{tabular}

Characteristic $p=23$, prime levels, dihedral

\begin{tabular}{||c|c|c|c|c|c|c|c|c|c||}
\hline Level & Wt & ResD & Dim & EmbDim & NilO & GorDef & \#Ops & $\#(\mathrm{p}<\mathrm{HB})$ & Gp \\
\hline 83 & 23 & 1 & 4 & 3 & 1 & 2 & 3 & 37 & $D_{3}$ \\
503 & 23 & 3 & 4 & 3 & 1 & 2 & 4 & 162 & $D_{7}$ \\
971 & 23 & 2 & 4 & 3 & 1 & 2 & 4 & 284 & $D_{5}$ \\
1259 & 23 & 1 & 4 & 3 & 1 & 2 & 3 & 358 & $D_{3}$ \\
\hline \hline
\end{tabular}

Characteristic $p=29$, prime levels, dihedral

\begin{tabular}{||c|c|c|c|c|c|c|c|c|c||}
\hline Level & Wt & ResD & Dim & EmbDim & NilO & GorDef & \#Ops & $\#(\mathrm{p}<\mathrm{HB})$ & $\mathrm{Gp}$ \\
\hline 107 & 29 & 1 & 4 & 3 & 1 & 2 & 3 & 55 & $D_{3}$ \\
199 & 29 & 1 & 4 & 3 & 1 & 2 & 3 & 92 & $D_{3}$ \\
\hline \hline
\end{tabular}

Characteristic $p=31$, prime levels, dihedral

\begin{tabular}{||c|c|c|c|c|c|c|c|c|c||}
\hline Level & Wt & ResD & Dim & EmbDim & NilO & GorDef & \#Ops & \#(p<HB $)$ & Gp \\
\hline 367 & 31 & 1 & 4 & 3 & 1 & 2 & 3 & 161 & $D_{3}$ \\
743 & 31 & 3 & 4 & 3 & 1 & 2 & 4 & 293 & $D_{7}$ \\
\hline \hline
\end{tabular}

Characteristic $p=37$, prime levels, dihedral

\begin{tabular}{||c|c|c|c|c|c|c|c|c|c||}
\hline Level & Wt & ResD & Dim & EmbDim & NilO & GorDef & \#Ops & $\#(\mathrm{p}<\mathrm{HB})$ & Gp \\
\hline 139 & 37 & 1 & 4 & 3 & 1 & 2 & 4 & 83 & $D_{3}$ \\
\hline \hline
\end{tabular}

Characteristic $p=41$, prime levels, dihedral

\begin{tabular}{||c|c|c|c|c|c|c|c|c|c||}
\hline Level & Wt & ResD & Dim & EmbDim & NilO & GorDef & \#Ops & \#(p<HB $)$ & Gp \\
\hline 83 & 41 & 1 & 4 & 3 & 1 & 2 & 3 & 61 & $D_{3}$ \\
139 & 41 & 1 & 4 & 3 & 1 & 2 & 4 & 92 & $D_{3}$ \\
431 & 41 & 1 & 4 & 3 & 1 & 2 & 5 & 233 & $D_{3}$ \\
\hline \hline
\end{tabular}

Characteristic $p=43$, prime levels, dihedral

\begin{tabular}{||c|c|c|c|c|c|c|c|c|c||}
\hline Level & Wt & ResD & Dim & EmbDim & NilO & GorDef & \#Ops & $\#(\mathrm{p}<\mathrm{HB})$ & Gp \\
\hline 419 & 43 & 1 & 4 & 3 & 1 & 2 & 3 & 239 & $D_{3}$ \\
\hline
\end{tabular}


Characteristic $p=47$, prime levels, dihedral

\begin{tabular}{||c|c|c|c|c|c|c|c|c|c||}
\hline Level & Wt & ResD & Dim & EmbDim & NilO & GorDef & \#Ops & $\#(\mathrm{p}<\mathrm{HB})$ & Gp \\
\hline 31 & 47 & 1 & 4 & 3 & 1 & 2 & 3 & 30 & $D_{3}$ \\
107 & 47 & 1 & 4 & 3 & 1 & 2 & 3 & 82 & $D_{3}$ \\
139 & 47 & 1 & 4 & 3 & 1 & 2 & 4 & 101 & $D_{3}$ \\
179 & 47 & 2 & 4 & 3 & 1 & 2 & 3 & 126 & $D_{5}$ \\
\hline \hline
\end{tabular}

Characteristic $p=53$, prime levels, dihedral

\begin{tabular}{||c|c|c|c|c|c|c|c|c|c||}
\hline Level & Wt & ResD & Dim & EmbDim & NilO & GorDef & \#Ops & $\#(\mathrm{p}<\mathrm{HB})$ & Gp \\
\hline 131 & 53 & 2 & 4 & 3 & 1 & 2 & 3 & 106 & $D_{5}$ \\
211 & 53 & 1 & 4 & 3 & 1 & 2 & 4 & 159 & $D_{3}$ \\
\hline \hline
\end{tabular}

Characteristic $p=59$, prime levels, dihedral

\begin{tabular}{||c|c|c|c|c|c|c|c|c|c||}
\hline Level & Wt & ResD & Dim & EmbDim & NilO & GorDef & \#Ops & $\#(\mathrm{p}<\mathrm{HB})$ & Gp \\
\hline 23 - & 59 & 1 & 4 & 3 & 1 & 2 & 4 & 30 & $D_{3}$ \\
211 & 59 & 1 & 4 & 3 & 1 & 2 & 4 & 175 & $D_{3}$ \\
227 & 59 & 1 & 4 & 3 & 1 & 2 & 3 & 187 & $D_{5}$ \\
227 & 59 & 1 & 4 & 3 & 1 & 2 & 3 & 187 & $D_{5}$ \\
367 & 59 & 1 & 4 & 3 & 1 & 2 & 3 & 279 & $D_{3}$ \\
\hline \hline
\end{tabular}

Characteristic $p=61$, prime levels, dihedral

\begin{tabular}{||c|c|c|c|c|c|c|c|c|c||}
\hline Level & Wt & ResD & Dim & EmbDim & NilO & GorDef & \#Ops & \#(p<HB $)$ & Gp \\
\hline 239 & 61 & 1 & 4 & 3 & 1 & 2 & 4 & 199 & $D_{5}$ \\
239 & 61 & 1 & 4 & 3 & 1 & 2 & 4 & 199 & $D_{5}$ \\
431 & 61 & 1 & 4 & 3 & 1 & 2 & 5 & 327 & $D_{3}$ \\
\hline \hline
\end{tabular}

Characteristic $p=67$, prime levels, dihedral

\begin{tabular}{||c|c|c|c|c|c|c|c|c|c||}
\hline Level & Wt & ResD & Dim & EmbDim & NilO & GorDef & \#Ops & $\#(\mathrm{p}<\mathrm{HB})$ & $\mathrm{Gp}$ \\
\hline 31 & 67 & 1 & 4 & 3 & 1 & 2 & 6 & 41 & $D_{3}$ \\
239 & 67 & 2 & 4 & 3 & 1 & 2 & 5 & 217 & $D_{5}$ \\
\hline \hline
\end{tabular}

Characteristic $p=71$, prime levels, dihedral

\footnotetext{
${ }^{5}$ First found by K. Buzzard, unpublished.
} 


\begin{tabular}{||c|c|c|c|c|c|c|c|c|c||}
\hline Level & Wt & ResD & Dim & EmbDim & NilO & GorDef & \#Ops & $\#(\mathrm{p}<\mathrm{HB})$ & $\mathrm{Gp}$ \\
\hline 59 & 71 & 1 & 4 & 3 & 1 & 2 & 3 & 71 & $D_{3}$ \\
239 & 71 & 1 & 4 & 3 & 1 & 2 & 5 & 223 & $D_{3}$ \\
283 & 71 & 1 & 4 & 3 & 1 & 2 & 5 & 263 & $D_{3}$ \\
\hline \hline
\end{tabular}

Characteristic $p=73$, prime levels, dihedral

\begin{tabular}{||c|c|c|c|c|c|c|c|c|c||}
\hline Level & Wt & ResD & Dim & EmbDim & NilO & GorDef & \#Ops & $\#(\mathrm{p}<\mathrm{HB})$ & $\mathrm{Gp}$ \\
\hline 211 & 73 & 1 & 4 & 3 & 1 & 2 & 4 & 209 & $D_{3}$ \\
283 & 73 & 1 & 4 & 3 & 1 & 2 & 5 & 269 & $D_{3}$ \\
\hline \hline
\end{tabular}

Characteristic $p=79$, prime levels, dihedral

\begin{tabular}{||c|c|c|c|c|c|c|c|c|c||}
\hline Level & Wt & ResD & Dim & EmbDim & NilO & GorDef & \#Ops & $\#(\mathrm{p}<\mathrm{HB})$ & Gp \\
\hline 307 & 79 & 1 & 4 & 3 & 1 & 2 & 5 & 307 & $D_{3}$ \\
\hline \hline
\end{tabular}

Characteristic $p=83$, prime levels, dihedral

\begin{tabular}{||c|c|c|c|c|c|c|c|c|c||}
\hline Level & Wt & ResD & Dim & EmbDim & NilO & GorDef & \#Ops & $\#(\mathrm{p}<\mathrm{HB})$ & Gp \\
\hline 47 & 83 & 2 & 4 & 3 & 1 & 2 & 4 & 67 & $D_{5}$ \\
79 & 83 & 2 & 4 & 3 & 1 & 2 & 3 & 101 & $D_{5}$ \\
107 & 83 & 1 & 6 & 3 & 2 & 2 & 3 & 132 & $D_{3}$ \\
211 & 83 & 1 & 4 & 3 & 1 & 2 & 4 & 232 & $D_{3}$ \\
251 & 83 & 1 & 4 & 3 & 1 & 2 & 3 & 271 & $D_{7}$ \\
251 & 83 & 1 & 4 & 3 & 1 & 2 & 3 & 271 & $D_{7}$ \\
251 & 83 & 1 & 4 & 3 & 1 & 2 & 3 & 271 & $D_{7}$ \\
\hline \hline
\end{tabular}

Characteristic $p=89$, prime levels, dihedral

\begin{tabular}{||c|c|c|c|c|c|c|c|c|c||}
\hline Level & Wt & ResD & Dim & EmbDim & NilO & GorDef & \#Ops & \#(p<HB $)$ & Gp \\
\hline 131 & 89 & 1 & 4 & 3 & 1 & 2 & 3 & 165 & $D_{5}$ \\
131 & 89 & 1 & 4 & 3 & 1 & 2 & 3 & 165 & $D_{5}$ \\
\hline \hline
\end{tabular}

Characteristic $p=97$, prime levels, dihedral

\begin{tabular}{||c|c|c|c|c|c|c|c|c|c||}
\hline Level & Wt & ResD & Dim & EmbDim & NilO & GorDef & $\#$ Ops & $\#(\mathrm{p}<\mathrm{HB})$ & Gp \\
\hline 307 & 97 & 1 & 4 & 3 & 1 & 2 & 5 & 367 & $D_{3}$ \\
\hline \hline
\end{tabular}

